\title{
W-shaped Profile and Breather-like Soliton of the Fractional Nonlinear Schrödinger Equation Describing the Polarization Mode in Optical Fibers
}

Alphonse HOUWE ( $\sim$ ahouw220@yahoo.fr)

University of Maroua: Universite de Maroua https://orcid.org/0000-0002-3619-8253

Souleymanou Abbagari

Universite de Maroua

Philippe Djorwe

Universite de Ngaoundere Faculte des Sciences

Youssoufa Saliou

University of Maroua: Universite de Maroua

Serge Yamigno Doka

Universite de Ngaoundere Faculte des Sciences

Mustafa Inc

Firat University: Firat Universitesi

\section{Research Article}

Keywords:

Posted Date: March 7th, 2022

DOI: https://doi.org/10.21203/rs.3.rs-1403677/v1

License: (1) This work is licensed under a Creative Commons Attribution 4.0 International License.

Read Full License 


\title{
W-shaped profile and Breather-like soliton of the Fractional Nonlinear Schrödinger Equation describing the Polarization Mode in Optical Fibers
}

\author{
Alphonse Houwe ${ }^{1,2 *}$, Souleymanou Abbagari ${ }^{3,4 *}$, Philippe Djorwe ${ }^{5}$, Youssoufa Saliou ${ }^{2}$, \\ Serge Y. Doka ${ }^{5 *}$, Mustafa $\operatorname{Inc}^{6,7,8}$ \\ ${ }^{1}$ Department of Marine Engineering, Limbe Nautical Arts and Fisheries Institute, P.O Box 485, Limbe, Cameroon. \\ ${ }^{2}$ Department of Physics, Faculty of Science, University of Maroua, P.O Box 814, Maroua, Cameroon. \\ ${ }^{3}$ Department of Basic Science, Faculty of Mines and Petroleum Industries, University of Maroua, P.O Box 08, \\ kaélé, Cameroon. \\ ${ }^{4}$ Laboratory of Mechanics, Materials and Structures, Department of physics, Faculty of Science, University of \\ Yaounde I, P.O Box 812, Yaoundé, Cameroon. \\ ${ }^{5}$ Department of Physics, Faculty of Science, University of Ngaoundere, P.O Box 454, Ngaoundere, Cameroon. \\ ${ }^{6}$ Department of Computer Engineering, Biruni University, Istanbul, Turkey \\ ${ }^{7}$ Department of Mathematics, Science Faculty, Firat University, 23119 Elazig, Turkey \\ ${ }^{8}$ Department of Medical Research, China Medical University Hospital, China Medical University, Taichung, Taiwan
}

\begin{abstract}
We use the nonlinear Schrödinger equation (NLSE) describing the polarization mode in optical fiber (OF) with Self-Steepening (SS), Self-Frequency Shift (SFS) and Cubic-quintic (CQ) term to analyze the effects of the fractional time parameter (FTP) on propagation of the breather-like solitons. We show the effects of the fractional parameter (FP) on the W-shaped profile, bright and dark optical soliton solutions as well as on the corresponding chirp component. It is observed that for small values of the FP, optical soliton shape is affected. Moreover, we show the effects of fraction time (FT) on Modulation Instability (MI) gain spectra. For all values of the FP, side lobes are formed and when these values increase the stability zones increases and the amplitude also increases values and the stability zones increases, both the stability zones and the soliton amplitude increase. We numerically investigate the propagation of the continuous waves $(\mathrm{CW})$. We show the formation of stable breather-like soliton for small values of the FTP.
\end{abstract}

\section{Introduction}

During the last past ten years, diverse research have been successfully emerged in different field of science such as biology, plasma, fluid mechanic, metamaterials, OF and so on [1 8 ]. One of the exact traveling waves

\footnotetext{
*Corresponding authors:,

E-mail address:

abbagaris@yahoo.fr (S. Abbagari).

ahouw220@yahoo.fr (A. Houwe).

E-mail: djorwepp@gmail.com (P. Djorwe).

ysaliou@yahoo.com(Youssoufa Saliou)

numami@gmail.com (S.Y. Doka).

minc@firat.edu.tr (M. Inc),.
} 
solutions most investigated during the last past years is solitary wave, called "soliton" which arise from interaction between nonlinearity and dispersion. Soliton can maintains its shape during propagation with a constant speed and can also conserves amplitude after collision with another soliton. Nowadays, soliton investigation has achieved the highest level in OF owing to the fact that many parameters are involved during its propagation namely Group velocity Dispersion (GVD), nonlinearity Kerr, Self-phase modulation(SPM), Cross-phase modulation (XPM) just to name a few [8 11]. It should be noted that solitons facilitated the transport of information, as well as the design of components serving to enhance the signal amplifier, stabilizer and sound level detectors during communication. Certainly, optical parameters effects on solitons were deeply investigated and a large variety of the nonlinear evolution equations (NLEE) which describe the propagation of pulse and ultrashort pulse in OF have been enrolled. We underline that NLEE with conformable derivative order (CFDO) are the most involved today owing to the fact that the conformable fractional derivative (CFD) parameter can play an important role during the propagation of soliton and on the well known aspect of the memory. Most often the NLSE with CFD order is used to stress analytical results in optical fibers. During the last decades, fractional differential equations have became important in diverse area of science. Several definitions of fractional derivatives have been pointed in many works, such as Grunwald-Letnikov definitions and Riemann-Liouville, Caputo, Atangana-Baleanu derivative in Caputo sense, Atangana-Baleanu fractional derivative in Riemann-Liouville sense and the well known CFD [12 14]. For example, in [15,16], it has been established the effects of the FT order on optical solitons and MI gain. The authors show for weak value of CFP, bright and dark optical soliton shaped are modified. More recently, in [17, they authors use the Chen Lee-Liu model with CF derivative time to figure out the importance of the fractional derivative on several analytical results. Many other works are concerned in literature to show the interesting features of the CFP, but just few have focussed on numerical investigation to fit with the predictions made on analytical investigation.

In this work, we aim to show the effects of the conformable time fractional (CTF) parameter [14] on exact traveling waves solution and optical soliton. We assume the dimensionless NLSE with CTF describing the polarization mode in OF. The model parameters are SS, SFS and the nonlinearities kerr, CQ in the presence of lower GVD. It has been shown by M. Eslami [18] and coworkers obtained analytical solutions such as chirped optical soliton. The model was also recently studied by Tala Tebue et al. [19] to investigate chirped soliton in OF. Bright, dark and cnoidal solitons are obtained. Out of this, several others works have been done in literature to find analytic exact traveling waves solutions by employing a huge class of mathematical methods such Auxiliary equation method, Rational method, $\left(G^{\prime} / G\right)$-Expansion Method, Fan-Extended Sub Equation (FESE) technic, Extended Modified Method, Modified Simple Equation Method, $\exp (-\varphi(q))$ Method, SubODE Method, Generalized Extended Algebraic Method (GEAM) and so on [1-11, 15, 20, 24, 40].

In our context, to examine the behavior of the analytic solutions and propagation of the $\mathrm{CW}$ in normal and anomalous regime, we use New Generalized Auxiliary Equation Method (NGAEM) and the linearizing technic. By varying the FP and fixed the values of the model, we establish how the shape of the W-shape profile and dark soliton are affected. Using the same procedure we show the variation of the MI gain. Through out numerical simulation, we establish the propagation of the breather-like soliton and chaotic breather-like soliton for certain values of the FTP. We organize our work as follows: in section 2 we summarize the 
NGAEM. In section 3, we apply the method and show the effects of the FTP on W-shaped, bright, dark optical soliton. The linearizing scheme is used in section 4 to establish the MI growth rate and set out the stable/unstable domains. To manage the robustness of the analytical results, we use numerical simulation in section 5 . We conclude the work in the last section.

\section{Methodology}

We stress the main steps of the NGAEM for finding traveling wave solutions of NLPDEs .

Step 1. Given a nonlinear physical model governed by partial differential equation

$$
G\left(\Phi, \Phi_{t}, \Phi_{z}, \Phi_{t t}, \Phi_{z z}, \ldots\right)=0
$$

Using the wave transformation $\Phi(z, t)=\Gamma(\xi), \xi=z-v t$, Eq.(1) is transformed into an ordinary differential equation (ODE) given by

$$
F\left(\Gamma, \Gamma^{\prime}, \Gamma^{\prime \prime}, \ldots\right)=0 .
$$

Step 2. Suppose that the solution of ODE Eq.(2) can be expressed as follows

$$
\Gamma(\xi)=\sum_{j=0}^{n} Y_{j}(\ell(\xi))^{j}
$$

where $Y_{j}(0 \leq j \leq n)$ are constant coefficients to be determined later and $\ell(\xi)$ satisfies the following auxiliary equations

$$
\begin{gathered}
\ell_{\xi}^{2}=2(\operatorname{Ln}(B))^{2} \times\left(H_{0}+H_{1} \ell(\xi)+H_{2} \ell(\xi)^{2}+H_{3} \ell(\xi)^{3}+H_{4} \ell(\xi)^{4}\right), \\
\ell_{\xi \xi}=(\operatorname{Ln}(B))^{2} \times\left(H_{1}+2 H_{2} \ell(\xi)+3 H_{3} \ell(\xi)^{2}+4 H_{4} \ell(\xi)^{3}\right) .
\end{gathered}
$$

Step 3: It is supposed that solution of Eq.(3) can be written in the following form

$$
\Gamma(\xi)=Y_{0}+Y_{1} \ell(\xi)+Y_{2} \ell(\xi)^{2}+Y_{3} \ell(\xi)^{3}+\ldots \ldots . Y_{n} \ell(\xi)^{n},
$$

where $Y_{0}, Y_{1}, Y_{3}, Y_{4}$ and $Y_{n}$ are reals constant to be determined later. Using the principle of the balance between the high-order nonlinear and derivative terms (2), we get the value of the integer $n$.

Step 4: With $n$ determined, we then collect all coefficients of powers of $\ell(\xi)$ in the resulting equation where these coefficients have to vanish. This will give a system of algebraic equations involving the parameters $Y_{0}$, $Y_{j}, j=(1,2, \ldots . ., n)$.

Step 5: To obtain the exact solutions of Eq.(2), the following solutions of Eq.(4) or Eq.(5) are used.

Case 1: for $H_{0}=H_{1}=H_{3}=0, H_{2}>0, H_{4}<0$,

$$
\ell(\xi)=\sqrt{\frac{-g h H_{2}}{H_{4}}} \operatorname{sech}_{B}\left(\sqrt{2 H_{2}} \xi\right)
$$

Case 2: for $H_{0}=\frac{H_{2}^{2}}{4 H_{4}}, H_{1}=H_{3}=0, H_{2}<0, H_{4}>0$,

$$
\ell(\xi)=\sqrt{\frac{-H_{2}}{2 H_{4}}} \tanh _{B}\left(\sqrt{-H_{2}} \xi\right) .
$$


Case 3: for $H_{0}=H_{1}=0, H_{2}>0, H_{4}>0, \tau= \pm 1$

$$
\ell(\xi)=\frac{g h H_{2} \operatorname{sech}_{B}^{2}\left(\sqrt{2 H_{2}} \frac{\xi}{2}\right)}{2 \tau \sqrt{H_{2} H_{4}} \tanh _{B}\left(\sqrt{2 H_{2}} \frac{\xi}{2}\right)-H_{3}} .
$$

Case 4: for $H_{0}=H_{1}=0, H_{2}>0, H_{3}^{2}-4 H_{2} H_{4}>0$,

$$
\ell(\xi)=\frac{2 \sqrt{g h} H_{2} \operatorname{sech}_{B}\left(\sqrt{2 H_{2}} \xi\right)}{\sqrt{H_{3}^{2}-4 H_{2} H_{4}}-\sqrt{g h} H_{3} \operatorname{sech}_{B}\left(\sqrt{2 H_{2}} \xi\right)} .
$$

Case 5: for $H_{0}=H_{1}=0, H_{2}>0, \tau= \pm 1$

$$
\ell(\xi)=\frac{g h H_{2} H_{3} \operatorname{sech}_{B}^{2}\left(\tau \sqrt{2 H_{2}} \frac{\xi}{2}\right)}{H_{2} H_{4}\left(1-\tanh _{B}\left(\tau \sqrt{2 H_{2}} \frac{\xi}{2}\right)\right)^{2}-H_{3}^{2}},
$$

where $H_{0}, H_{1}, H_{2}, H_{3}$ and $H_{4}$ are arbitrary constants. Therefore, using Eqs.(7-11) and Eq.(3) or Eq.(6), the exact solutions to Eq.(2) can be obtained.

The generalized hyperbolic and triangular functions are defined as

$$
\begin{array}{llll}
\sinh _{B}(\xi) & =\frac{g B^{\xi}-h B^{-\xi}}{2}, & \cosh _{B}(\xi)=\frac{g B^{\xi}+h B^{-\xi}}{2}, & \tanh _{B}(\xi)=\frac{g B^{\xi}-h B^{-\xi}}{g B^{\xi}+h B^{-\xi}}, \\
\operatorname{coth}_{B}(\xi)=\frac{g B^{\xi}+h B^{-\xi}}{g B^{\xi}-h B^{-\xi}}, & \operatorname{sech}_{B}(\xi)=\frac{2}{g B^{\xi}+h B^{-\xi}}, & \operatorname{csch}_{B}(\xi)=\frac{2}{g B^{\xi}-h B^{-\xi}}, \\
\sin _{B}(\xi)=\frac{g B^{i \xi}-h B^{-i \xi}}{2 i}, & \cos _{B}(\xi)=\frac{g B^{i \xi}+h B^{-i \xi}}{2}, & \tan _{B}(\xi)=-i \frac{g B^{i \xi}-h B^{-i \xi}}{g B^{i \xi}+h B^{-i \xi}}, \\
\cot _{B}(\xi)=i \frac{g B^{i \xi}+h B^{-i \xi}}{g B^{i \xi}-h B^{-i \xi}}, & \sec _{B}(\xi)=\frac{2}{g B^{i \xi}+h B^{-i \xi}}, & \csc _{B}(\xi)=\frac{2 i}{g B^{i \xi}-h B^{-i \xi}}
\end{array}
$$

where $\xi$ is an independent variable, the constants $g>0$ and $h>0$ are called deformation parameters.

\subsection{Effects of the fractional parameter on soliton}

We aim in this Section, to examine the effects of the CFTP and the physical parameters (PP) of NLSE with the conformable time fractional which describes the polarization mode on analytical and numerical chirp and chirped soliton. Sundry prospective definitions of the fractional derivative have been obtained and more details of the conformable fractional derivative properties are given in [14 21-23]. For analytical investigation, we use the NGAEM integration algorithm. To consolidate the analytical investigation, we employ the Splitstep Fourier and Runge Kutta algorithm to show step by step the effects of the FP associate with the PP of the model. It is worth to indicate that the the NLSE describing the polarization mode has been recently handle analytically by Eslami and coworkers [18]. Chirped optical soliton have been studied by using the rational method. The authors show the effects of several parameters of the model on the chirp and the corresponding chirped optical soliton. More after in ref. [20], significant results on chirped femtosecond solitons and kinklike solitons have been pointed out. The figure of merit of SS and SFS parameters were highlighted. The governing equation with FTP which describing the polarization mode is set as follows [18, 19]: 


$$
\begin{aligned}
& i \frac{\partial \psi(z, t)}{\partial z}+\alpha D_{t}^{2 \beta} \psi(z, t)+s\left(|\psi(z, t)|^{2} \psi(z, t)\right)+i \gamma D_{t}^{\beta}\left(|\psi(z, t)|^{2} \psi(z, t)\right)+i \epsilon \psi(z, t) D_{t}^{\beta}\left(|\psi(z, t)|^{2}\right) \\
& +\theta|\psi(z, t)|^{4} \psi(z, t)=0, \quad 0<\beta \leq 1 .
\end{aligned}
$$

The quantity $\psi(z, t)$ is the complex polarization mode amplitude, while the GVD coefficient is $\alpha$, SS term and SFS are respectively $\gamma$ and $\epsilon$. However, $z$ is the space parameter and $t$ the delayed time. The parameter $s$ is real and denotes the kerr nonlinear coefficient. In addition, the nonlinear coefficient which counts for CQ nonlinearity is denoted by $\theta$. Some analytic investigation have been recently done by using the model [18,19]. They authors have established the existence of chirp components and Chirped solitons as solutions. These results have certainly open the way to another analytic investigation, but in our knowledge no more works have been done to confirm the stability of these results. As the mathematical model contains nonlinear terms and weak dispersion coefficient, we employ tiny perturbations of the CW as solution of Eq.(12) to analyze the MI spectra with the effects of the FP. Assuming the Riemann-Liouvile form [21-23], we assume the following transformation with the chirp components as follows:

$$
\psi(z, t)=L(\xi) e^{i(C(\xi)-\kappa z)},
$$

and $\xi=\frac{t^{\beta}}{\beta}-u z$, where $u=\frac{1}{\vartheta}, \vartheta$ denotes the soliton speed. The chirp component can be expressed as

$$
\delta \omega(z, t)=-\frac{\partial}{\partial z}(C(\xi)-\kappa z)=\frac{1}{\vartheta} C_{\xi}=0 .
$$

We transform Eq.(12) to the ODE by using Eq.(13), we obtain respectively, the real and imaginary components, having the form,

$$
\begin{aligned}
& \alpha L_{\xi \xi}+\kappa L+s L^{3}-\gamma C_{\xi} L^{3}-\alpha L\left(C_{\xi}\right)^{2}+u L C_{\xi}+\theta L^{5}=0, \\
& u L_{\xi}-(3 \gamma+2 \epsilon) L_{\xi} L^{2}-2 \alpha L_{\xi} C_{\xi}-\alpha C_{\xi \xi} L=0 .
\end{aligned}
$$

Now, we extract the chirp component by multiplying Eq.(16) by $L$ and then integrating once, we show the following equation in terms of $L^{2}$

$$
C_{\xi}=-\frac{(3 \gamma+2 \epsilon)}{4 \alpha} L^{2}+\frac{1}{2 \alpha \vartheta}
$$

and the corresponding chirp parameters gives

$$
\delta \omega(z, t)=-\frac{(3 \gamma+2 \epsilon)}{4 \alpha \vartheta} L^{2}+\frac{1}{2 \alpha \vartheta^{2}} .
$$

It results that the chirp components depends on the GVD coefficient, SS and the SFS parameters. We can insert Eq.(17) into Eq.(15) and the ODE in the elliptic form is obtained as

$$
L_{\xi \xi}+\left(\frac{4 \alpha \kappa+u^{2}}{4 \alpha^{2}}\right) L+\left(\frac{2 \alpha s-\gamma u}{2 \alpha^{2}}\right) L^{3}\left(\frac{16 \alpha \theta-4 \epsilon^{2}-4 \epsilon \gamma+3 \gamma^{2}}{16 \alpha^{2}}\right) L^{5}=0 .
$$


We adopt the transformation $L(\xi)=\sqrt{E(\xi)}$ to reduce Eq.(19) to the integrable form as

$$
\left(E_{\xi}\right)^{2}-\left(\frac{4 \alpha \kappa+u^{2}}{4 \alpha^{2}}\right) E^{2}-2\left(\frac{2 \alpha s-\gamma u}{2 \alpha^{2}}\right) E^{3}-4\left(\frac{16 \alpha \theta-4 \epsilon^{2}-4 \epsilon \gamma+3 \gamma^{2}}{16 \alpha^{2}}\right) E^{4}-2 E E_{\xi \xi}=0 .
$$

Now, we use the homogeneous balanced principle between $E E_{\xi \xi}$ and $E^{4}$ in Eq. (20), which leads to $n=1$. Subsequently, it is introduced into Eq.(3) or Eq.(6) the value of $n$, and taking into account Eqs.(4-5), a system of equation in terms of $\ell(\xi)$ is obtained. Thus, we set all the coefficients of each individual term $\ell(\xi)$ to zero, which gives the results below.

\section{- Set 1}

$$
\begin{aligned}
& A_{0}=0, A_{1}=\sqrt{\frac{-24 H_{4}(\alpha \operatorname{Ln}(B))^{2}}{16 \alpha \theta-4 \epsilon^{2}-4 \epsilon \gamma+3 \gamma^{2}}}, H_{2}=\frac{-2\left(\alpha s^{2}+\gamma^{2} \kappa\right)}{(\operatorname{Ln}(B))^{2} \gamma^{2} \alpha}, u=\frac{2 \alpha s}{\gamma}, \\
& H_{4}=H_{4}, \theta=\theta
\end{aligned}
$$

with $-24 H_{4}\left(16 \alpha \theta-4 \epsilon^{2}-4 \epsilon \gamma+3 \gamma^{2}\right)>0$ and $\gamma \neq 0, \alpha \neq 0$.

$$
\begin{aligned}
& A_{0}=\frac{4}{5} \frac{4 \kappa \alpha+u^{2}}{(-2 \alpha s+\gamma u)}, H_{2}=\frac{1}{10} \frac{4 \kappa \alpha+u^{2}}{\alpha^{2}(\operatorname{Ln}(B))^{2}}, H_{4}=-\frac{5 A_{1}^{2}(-2 \alpha s+(\gamma) u)^{2}}{\left(128 \kappa \alpha+32 u^{2}\right) \alpha^{2}(\operatorname{Ln}(B))^{2}} \\
& \theta=-\frac{-60 \alpha^{2} s^{2}-64 \alpha \epsilon^{2} \kappa-64 \alpha \epsilon \gamma \kappa+48 \alpha \gamma^{2} \kappa+60 \alpha \gamma s u-16 \epsilon^{2} u^{2}-16 \epsilon \gamma u^{2}-3 \gamma^{2} u^{2}}{\left(256 \kappa \alpha+64 u^{2}\right) \alpha} \\
& A_{1}=A_{1}, u=u
\end{aligned}
$$

with the constraint condition as $(-2 \alpha s+\gamma u) \neq 0,\left(128 \kappa \alpha+32 u^{2}\right) \alpha^{2} \neq 0$,

- Case 1: For $H_{0}=H_{1}=H_{3}=0$, and $H_{2}>0, H_{4}<0$ using Eqs.(21-22) respectively, the bright soliton in the governing model (12) is acquired as

$$
\begin{aligned}
& \psi_{1,1,1}(z, t)=\sqrt{A_{1} \sqrt{\frac{-g h H_{2}}{H_{4}}} \operatorname{sech}_{B}\left(\sqrt{2 H_{2}}\left(\frac{t^{\beta}}{\beta}-\frac{2 \alpha s}{\gamma} z\right)\right) e^{i\left(C\left(\frac{t^{\beta}}{\beta}-\frac{2 \alpha s}{\gamma} z\right)-\kappa z\right),}} \\
& \psi_{1,1,2}(z, t)=\sqrt{A_{0}+A_{1} \sqrt{\frac{-g h H_{2}}{H_{4}}} \operatorname{sech}_{B}\left(\sqrt{2 H_{2}}\left(\frac{t^{\beta}}{\beta}-u z\right)\right) e^{i\left(C\left(\frac{t^{\beta}}{\beta}-u z\right)-\kappa z\right)},}
\end{aligned}
$$

and the corresponding chirp gives

$$
\begin{aligned}
& \delta \omega_{1,1,1}(z, t)=-\frac{(3 \gamma+2 \epsilon) A_{1}}{4 \alpha \vartheta} \sqrt{\frac{-g h H_{2}}{H_{4}}} \operatorname{sech}_{B}\left(\sqrt{2 H_{2}}\left(\frac{t^{\beta}}{\beta}-\frac{2 \alpha s}{\gamma} z\right)\right)+\frac{1}{2 \alpha \vartheta^{2}} \\
& \delta \omega_{1,1,2}(z, t)=-\frac{(3 \gamma+2 \epsilon)}{4 \alpha \vartheta}\left[A_{0}+A_{1} \sqrt{\frac{-g h H_{2}}{H_{4}}} \operatorname{sech}_{B}\left(\sqrt{2 H_{2}}\left(\frac{t^{\beta}}{\beta}-\frac{2 \alpha s}{\gamma} z\right)\right)\right]+\frac{1}{2 \alpha \vartheta^{2}} .
\end{aligned}
$$

In the plotted figures 1, 2 and 3 we show the effects of the conformable derivative time fractional $(\mathrm{CDTF})$ order and fixed values of OF parameters $(\alpha=0.1, \epsilon=-0.02, s=0.25, \theta=0.1)$ on the contour plot evolution (figure 1 ( $a, b, c, d, e, f)$ ), W-shaped profile (figure 2 ( $a, b, c, d)$ ) and spatial evolution of the bright optical soliton (figure 3). We observed the propagation of soliton with the variation of the FTP. For $\beta=0.45$ the width of $\mathrm{W}$-shaped is important between $10 \leq t \leq 20$ (see figure $1(\mathrm{a}, \mathrm{b}))$. When we increase the value of the CFP to $\beta=0.65, \beta=0.75, \beta=0.85$ and $\beta=0.95$ illustrated through figure $1(\mathrm{c}, \mathrm{b}, \mathrm{d}, \mathrm{e}, \mathrm{f})$, it is observed that the width of the $\mathrm{W}$-shaped 
shrinks and takes the valued of $t=10$. Moreover, we have shown the impact of the CFP on the evolution of $\mathrm{W}$-shaped profile in figure 2. For $\beta=0.6$ the width of the $\mathrm{W}$-shaped increases and the width shrinks when the value of the CFP increases (see figure $2(b, c, d)$ ). In figure 3 , we set out once again the clout of the CFP on W-shaped profile. For small value of the CFP, there is modification on the width of the $\mathrm{W}$-shaped which seem to propagate in the direction of the red arrow (see figure $3(\mathrm{a}, \mathrm{b}, \mathrm{c})$ ). When we increase the value of the CFP, it appears that the width of the $\mathrm{W}$-shaped profile shrinks. Beside, in plot figure 4 we show the evolution of the bright optical soliton with fixed value of the model parameters and fixed valued of the CFP $(\beta=0.25)$ with the variation of times. We also, noticed the effects of the CFP on the corresponding chirp for $\beta=0.2$ in figure $5(\mathrm{a}, \mathrm{b})$ and $\beta=0.4$ in figure $5(\mathrm{c}, \mathrm{d})$. We observe a stretching of the amplitude of the chirp bright which seems to dissect according to the direction of the red arrow during propagation (see figure $5(\mathrm{a}, \mathrm{b})$ ). In plot figure $5(\mathrm{c}, \mathrm{d})$ the red circle shows the fragments of the chirp caused by the effects of the FTP. These fragments indicate the dispersion of wave energy during propagation of the corresponding chirp signal. Considering now the constraint condition $H_{2}<0$ and $H_{4}>0$, we plot in figure 6 the behavior of the bright optical soliton with the effects of the FTP and fixed values of the model parameters (MP). It is observed that when the value of the CFP increases the bright optical soliton shape is well formed and shift from left to right by maintaining without any deformation. In figure 7 with set the constraint condition $A_{0} \neq 0$, $\alpha \neq 0, H_{4}>0$ and $H_{2}>0$ we want to show the breather like soliton evolution under the effects of the CFP. We increase the value of the CFP and we see that the obtained breather increases in amplitude (see figure $7(\mathrm{~b}, \mathrm{c})$ ), which denotes a gain of energy during propagation.

This scenario explain energy transfer caused by the effects of CFP during propagation of W-shaped and breather like soliton in OF. These results could give new opportunities to data processing and communication improvement. Compared these results with refs. [18 20, news behavior of the $\mathrm{W}$-shaped profile are obtained when the valued of the FT parameter decreases.

- Case 2: For $H_{0}=\frac{H_{2}^{2}}{4 H_{4}}, H_{1}=H_{3}=0$, and $H_{2}<0, H_{4}>0$, using Eqs.(21-22) respectively, the dark soliton is revealed as

$$
\begin{aligned}
& \psi_{1,2,1}(z, t)=\sqrt{\left.A_{1} \sqrt{\frac{-H_{2}}{2 H_{4}}} \tanh _{B}\left(\sqrt{-H_{2}}\left(\frac{t^{\beta}}{\beta}-\frac{2 \alpha s}{\gamma} z\right)\right)\right) e^{i\left(C\left(\frac{t^{\beta}}{\beta}-\frac{2 \alpha s}{\gamma} z\right)-\kappa z\right)},} \\
& \psi_{1,2,2}(z, t)=\sqrt{\left.A_{0}+A_{1} \sqrt{\frac{-H_{2}}{2 H_{4}}} \tanh _{B}\left(\sqrt{-H_{2}}\left(\frac{t^{\beta}}{\beta}-u z\right)\right)\right) e^{i\left(C\left(\frac{t^{\beta}}{\beta}-u z\right)-\kappa z\right)}}
\end{aligned}
$$

the corresponding chirp expression

$$
\begin{aligned}
& \left.\delta \omega_{1,2,1}(z, t)=-\frac{(3 \gamma+2 \epsilon) A_{1}}{4 \alpha \vartheta} \sqrt{\frac{-H_{2}}{2 H_{4}}} \tanh _{B}\left(\sqrt{-H_{2}}\left(\frac{t^{\beta}}{\beta}-\frac{2 \alpha s}{\gamma} z\right)\right)\right)+\frac{1}{2 \alpha \vartheta^{2}} \\
& \left.\delta \omega_{1,2,2}(z, t)=-\frac{(3 \gamma+2 \epsilon)}{4 \alpha \vartheta}\left[A_{0}+A_{1} \sqrt{\frac{-H_{2}}{2 H_{4}}} \tanh _{B}\left(\sqrt{-H_{2}}\left(\frac{t^{\beta}}{\beta}-u z\right)\right)\right)\right]+\frac{1}{2 \alpha \vartheta^{2}} .
\end{aligned}
$$



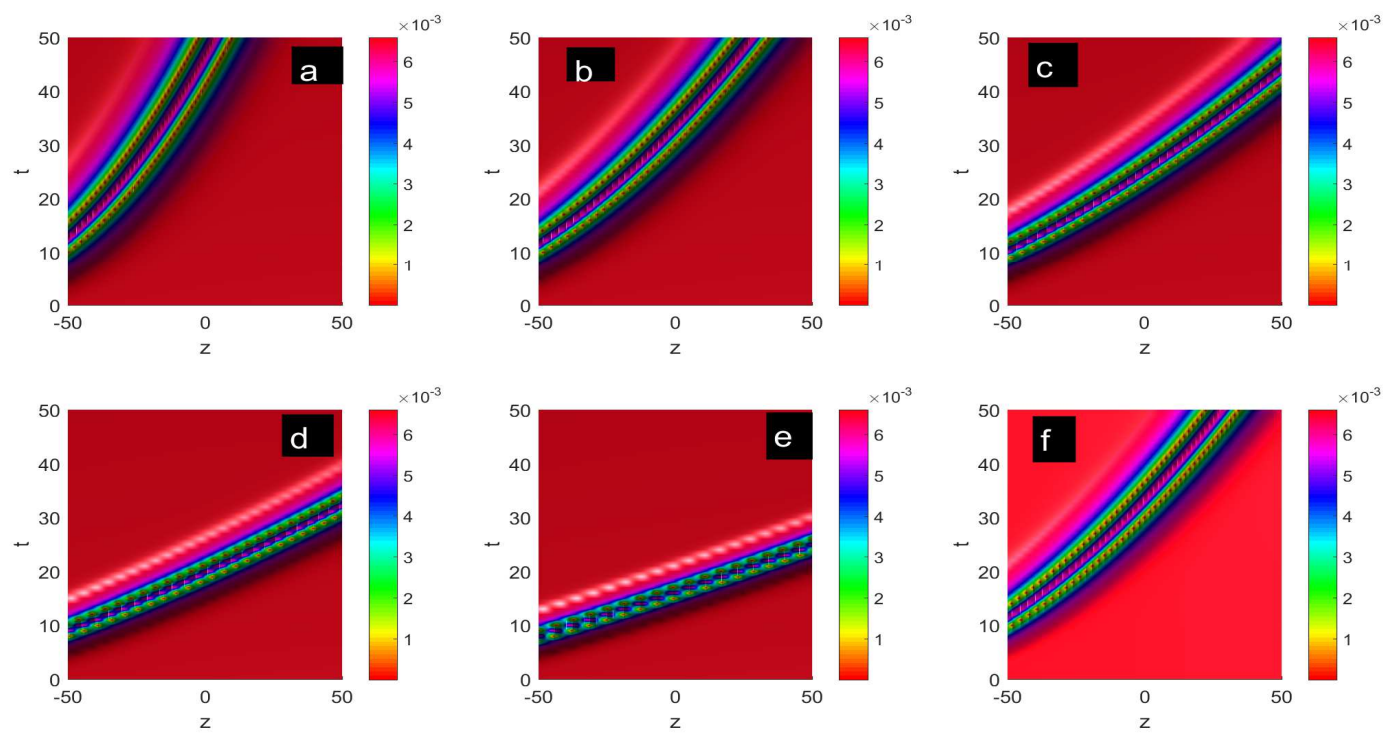

Figure 1: Contour evolution of W-shaped profile soliton $\left|\psi_{1,1,2}(z, t)\right|^{2}(\mathrm{a})[\beta=0.45]$, (b) $[\beta=0.55]$, (c) $[\beta=$ 0.65], (d)[ $\beta=0.75]$, (e)[ $\beta=0.85]$, (f)[ $\beta=0.95]$ at $B=e, \alpha=0.1, g=0.042, h=0.00078, \epsilon=-0.02, s=$ $0.25, H_{4}=-0.095, \theta=0.1, H_{2}=1.84, A_{0}=-0.06, A_{1}=0.5$
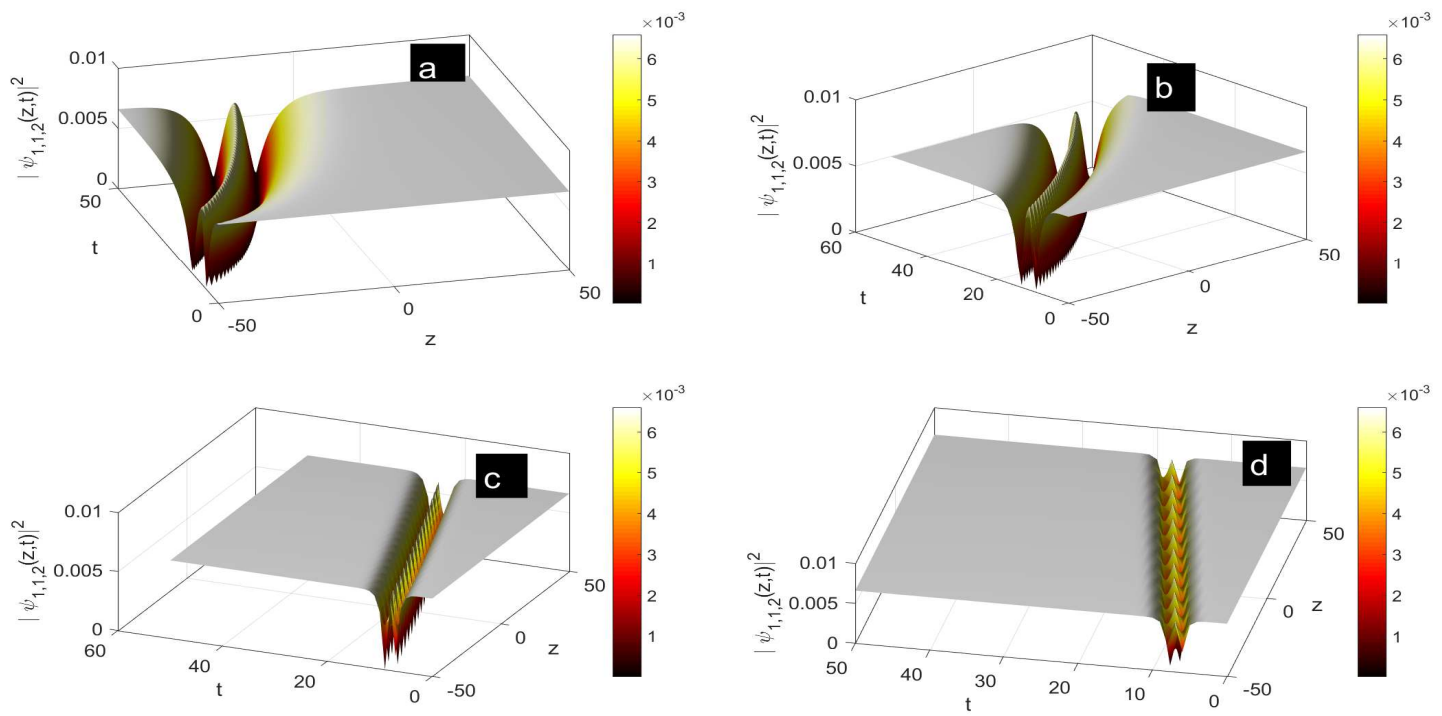

Figure 2: Evolution of W-shaped profile soliton $\left|\psi_{1,1,2}(z, t)\right|^{2}$ with the effects of the CFP ( $\beta$ ) (a)[ $\left.\beta=0.6\right]$, (b) $[\beta=0.7],(\mathrm{c})[\beta=0.8],(\mathrm{d})[\beta=1]$ at $B=e, \alpha=0.1, g=0.042, h=0.00078, \epsilon=-0.02, s=0.25, H_{4}=$ $-0.095, \theta=0.1, H_{2}=1.84, A_{0}=-0.06, A_{1}=0.5$ 

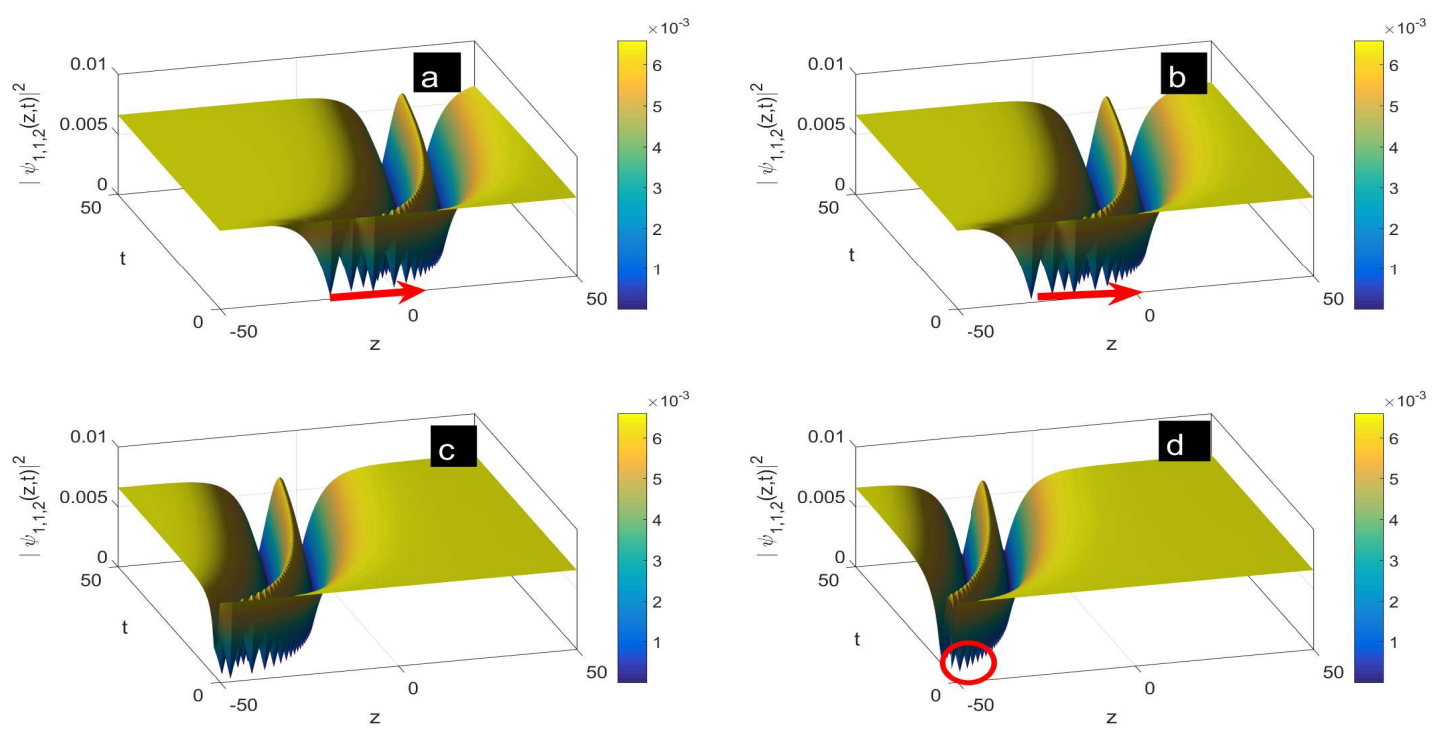

Figure 3: Evolution of W-shaped profile soliton $\left|\psi_{1,1,2}(z, t)\right|^{2}$ with the effects of the tiny CFP ( $\beta$ ) (a)[ $\beta=$ 0.09], (b)[ $\beta=0.1]$, (c) $[\beta=0.15],(\mathrm{d})[\beta=0.2]$ at $B=e, \alpha=0.1, g=0.042, h=0.00078, \epsilon=-0.02, s=$ $0.25, H_{4}=-0.095, \theta=0.1, H_{2}=1.84, A_{0}=-0.06, A_{1}=0.5$
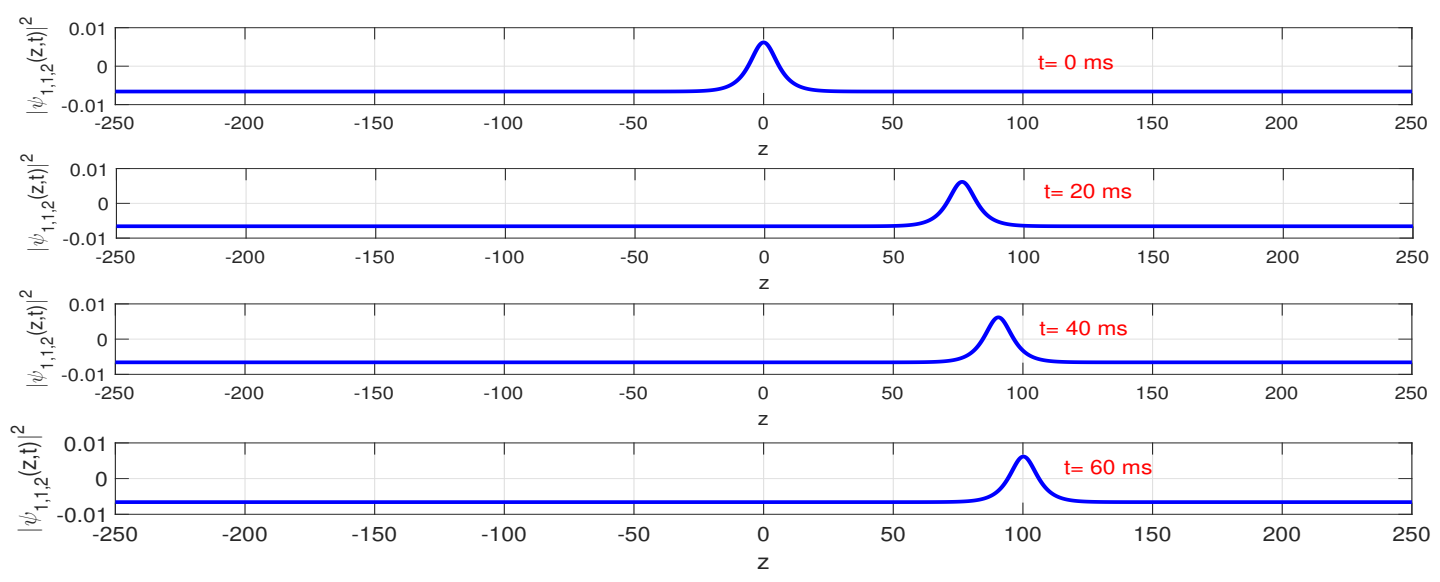

Figure 4: Evolution of bright soliton $\left|\psi_{1,1,2}(z, t)\right|^{2}$ with the effects of the CFP $(\beta=0.25)$ at $B=e, \alpha=$ $0.1, g=0.042, h=0.00078, \epsilon=-0.02, s=0.25, H_{4}=-0.095, \theta=0.1, H_{2}=1.84, A_{0}=-0.06, A_{1}=0.5$ 

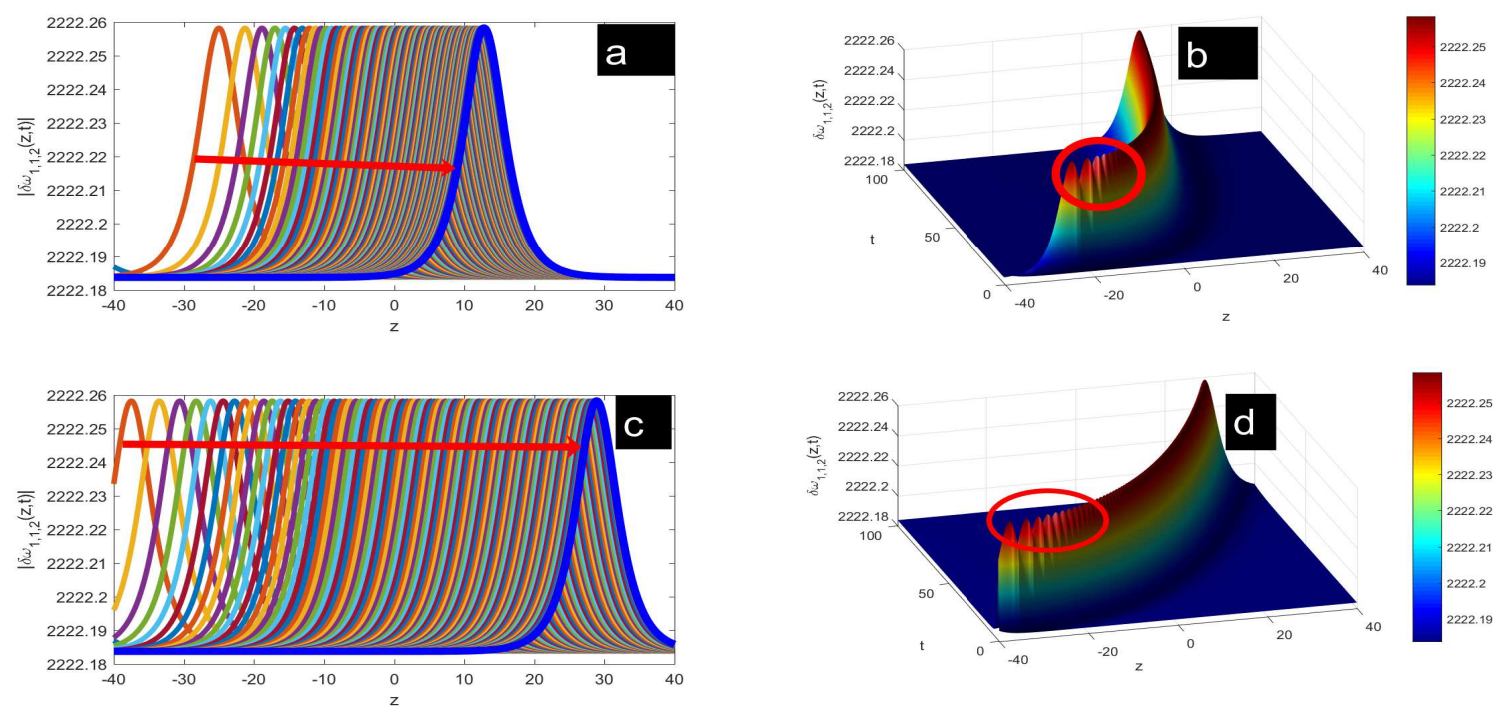

Figure 5: Chirp evolution $\left|\delta \omega_{1,1,2}(z, t)\right|$ with the effects of the CFP $(\beta=0.25)$ at $B=e, \alpha=0.1, g=$ $0.042, h=0.00078, \epsilon=-0.02, s=0.25, H_{4}=-0.095, \theta=0.1, H_{2}=1.84, \gamma=0.045, A_{0}=-0.06, A_{1}=$ 0.5
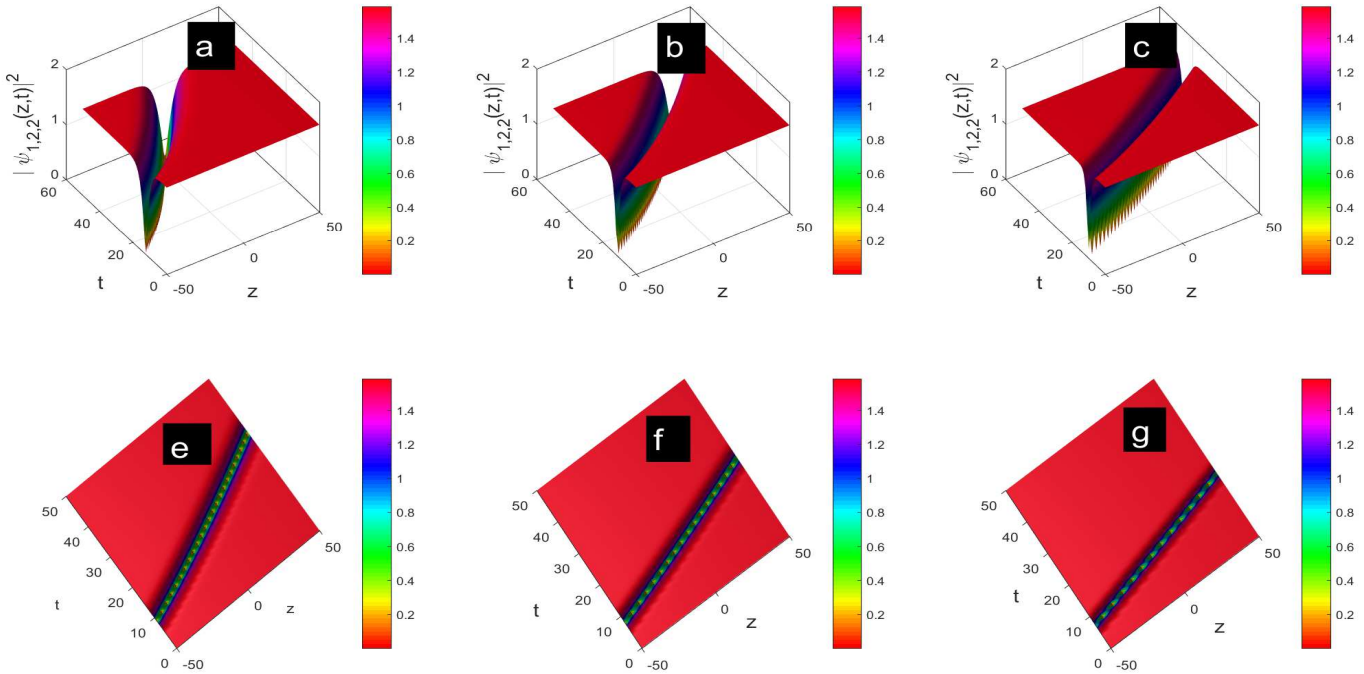

Figure 6: Dark optical soliton evolution $\left|\delta \psi_{1,2,2}(z, t)\right|^{2}$ with the effects of the CFP (a) $[\beta=0.45]$, (b) $[\beta=0.55]$, (c) $[\beta=0.65]$, (d) $[\beta=0.65]$, (e) $[\beta=0.75]$, (f) $[\beta=0.85]$ and (g) $[\beta=0.95]$ at $B=e, \alpha=$ $0.01, \epsilon=-0.02, s=0.25, H_{4}=0.095, \theta=0.1, H_{2}=-1.84, \gamma=0.045, A_{0}=-0.06, A_{1}=0.5$ 
- Set 2

$$
\begin{gathered}
A_{0}=0, A_{1}=A_{1}, H_{2}=-\frac{1}{2} \frac{4 \alpha \kappa+u^{2}}{\alpha^{2}(\operatorname{Ln}(B))^{2}}, H_{3}=\frac{1}{2} \frac{A_{1}(-2 \alpha s+\gamma u)}{\alpha^{2}(\operatorname{Ln}(B))^{2}}, \theta=\theta, u=u, \\
H_{4}=-\frac{1}{24} \frac{A_{1}^{2}\left(16 \alpha \theta-4 \epsilon^{2}-4 \epsilon \gamma+3 \gamma^{2}\right)}{\alpha^{2}(\operatorname{Ln}(B))^{2}}, \\
A_{0}=A_{0}, A_{1}=A_{1}, H_{2}=-\frac{1}{2} \frac{-2 \alpha s A_{0}+\gamma u A_{0}-4 \alpha \kappa-u^{2}}{\alpha^{2}(\operatorname{Ln}(B))^{2}}, u=u, \\
H_{3}=\frac{A_{1}\left(10 \alpha s A_{0}-5 \gamma u A_{0}+16 \alpha \kappa+4 u^{2}\right)}{6 \alpha^{2}(\operatorname{Ln}(B))^{2} A_{0}}, H_{4}=-\frac{1}{6} \frac{A_{1}^{2}\left(-4 \alpha s A_{0}+2 \gamma u A_{0}-4 \alpha \kappa-u^{2}\right)}{A_{0}^{2} \alpha^{2}(\operatorname{Ln}(B))^{2}}, \\
\theta=-\frac{1}{16} \frac{-4 \epsilon^{2} A_{0}^{2}-4 \epsilon \gamma A_{0}^{2}+3 \gamma^{2} A_{0}^{2}+16 \alpha s A_{0}-8 \gamma u A_{0}+16 \alpha \kappa+4 u^{2}}{\alpha A_{0}^{2}},
\end{gathered}
$$

- Case 3: For $H_{0}=H_{1}=0, H_{2}>0 H_{4}>0, \tau= \pm 1$ using Eqs.(31-32) respectively, is revealed as

$$
\begin{aligned}
& \psi_{2,3,1}(z, t)=\sqrt{\frac{g h A_{1} H_{2} \operatorname{sech}_{B}^{2}\left(\sqrt{2 H_{2}} \frac{\left(\frac{t^{\beta}}{\beta}-u z\right)}{2}\right)}{2 \tau \sqrt{H_{2} H_{4}} \tanh _{B}\left(\sqrt{2 H_{2}} \frac{\left(\frac{t^{\beta}}{\beta}-u z\right)}{2}\right)-H_{3}} e^{i\left(C\left(\frac{t^{\beta}}{\beta}-u z\right)-\kappa z\right),}} \\
& \psi_{2,3,2}(z, t)=\sqrt{A_{0}+\frac{g h A_{1} H_{2} \operatorname{sech}_{B}^{2}\left(\sqrt{2 H_{2}} \frac{\left(\frac{t^{\beta}}{\beta}-u z\right)}{2}\right)}{2 \tau \sqrt{H_{2} H_{4}} \tanh _{B}\left(\sqrt{2 H_{2}} \frac{\left(\frac{t^{\beta}}{\beta}-u z\right)}{2}\right)-H_{3}} e^{i\left(C\left(\frac{t^{\beta}}{\beta}-u z\right)-\kappa z\right),}}
\end{aligned}
$$

thereafter the corresponding chirp components gives

- Case 3: For $H_{0}=H_{1}=0, H_{2}>0 H_{4}>0, \tau= \pm 1$ using Eqs.(31-32) respectively, is revealed as

$$
\begin{aligned}
& \delta \omega_{2,3,1}(z, t)=-\frac{(3 \gamma+2 \epsilon)}{4 \alpha \vartheta} \frac{g h A_{1} H_{2} \operatorname{sech}_{B}^{2}\left(\sqrt{2 H_{2}} \frac{\left(\frac{t^{\beta}}{\beta}-u z\right)}{2}\right)}{2 \tau \sqrt{H_{2} H_{4}} \tanh _{B}\left(\sqrt{2 H_{2}} \frac{\left(\frac{t^{\beta}}{\beta}-u z\right)}{2}\right)-H_{3}}+\frac{1}{2 \alpha \vartheta^{2}}, \\
& \delta \omega_{2,3,2}(z, t)=-\frac{(3 \gamma+2 \epsilon)}{4 \alpha \vartheta}\left[A_{0}+\frac{g h A_{1} H_{2} \operatorname{sech}_{B}^{2}\left(\sqrt{2 H_{2}} \frac{\left(\frac{t^{\beta}}{\beta}-u z\right)}{2}\right)}{2 \tau \sqrt{H_{2} H_{4}} \tanh _{B}\left(\sqrt{2 H_{2}} \frac{\left(\frac{t^{\beta}}{\beta}-u z\right)}{2}\right)-H_{3}}\right]+\frac{1}{2 \alpha \vartheta^{2}} .
\end{aligned}
$$

- Case 4: For $H_{0}=H_{1}=0$, and $H_{2}>0, H_{3}{ }^{2}-4 H_{2} H_{4}>0$, using Eqs.(31-32) respectively, is revealed as

$$
\begin{aligned}
& \psi_{2,4,1}(z, t)=\sqrt{\frac{2 \sqrt{g h} A_{1} H_{2} \operatorname{sech}_{B}\left(\sqrt{2 H_{2}}\left(\frac{t^{\beta}}{\beta}-u z\right)\right)}{\sqrt{H_{3}^{2}-4 H_{2} H_{4}}-\sqrt{g h} H_{3} \operatorname{sech}_{B}\left(\sqrt{2 H_{2}}\left(\frac{t^{\beta}}{\beta}-u z\right)\right)} e^{i\left(C\left(\frac{t^{\beta}}{\beta}-u z\right)-\kappa z\right),}} \\
& \psi_{2,4,2}(z, t)=\sqrt{A_{0}+\frac{2 \sqrt{g h} A_{1} H_{2} \operatorname{sech}_{B}\left(\sqrt{2 H_{2}}\left(\frac{t^{\beta}}{\beta}-u z\right)\right)}{\sqrt{H_{3}^{2}-4 H_{2} H_{4}}-\sqrt{r s} H_{3} \operatorname{sech}_{B}\left(\sqrt{2 H_{2}}\left(\frac{t^{\beta}}{\beta}-u z\right)\right)} e^{i\left(C\left(\frac{t^{\beta}}{\beta}-u z\right)-\kappa z\right),}}
\end{aligned}
$$

and the corresponding chirp gives

$$
\begin{aligned}
& \delta \omega_{2,4,1}(z, t)=-\frac{(3 \gamma+2 \epsilon)}{4 \alpha \vartheta} \frac{2 \sqrt{g h} A_{1} H_{2} \operatorname{sech}_{B}\left(\sqrt{2 H_{2}}\left(\frac{t^{\beta}}{\beta}-u z\right)\right)}{\sqrt{H_{3}^{2}-4 H_{2} H_{4}}-\sqrt{g h} H_{3} \operatorname{sech}_{B}\left(\sqrt{2 H_{2}}\left(\frac{t^{\beta}}{\beta}-u z\right)\right)}+\frac{1}{2 \alpha \vartheta^{2}}, \\
& \delta \omega_{2,4,2}(z, t)=-\frac{(3 \gamma+2 \epsilon)}{4 \alpha \vartheta}\left[A_{0}+\frac{2 \sqrt{g h} A_{1} H_{2} \operatorname{sech}_{B}\left(\sqrt{2 H_{2}}\left(\frac{t^{\beta}}{\beta}-u z\right)\right)}{\sqrt{H_{3}^{2}-4 H_{2} H_{4}}-\sqrt{r s} H_{3} \operatorname{sech}_{B}\left(\sqrt{2 H_{2}}\left(\frac{t^{\beta}}{\beta}-u z\right)\right)}\right]+\frac{1}{2 \alpha \vartheta^{2}} .
\end{aligned}
$$



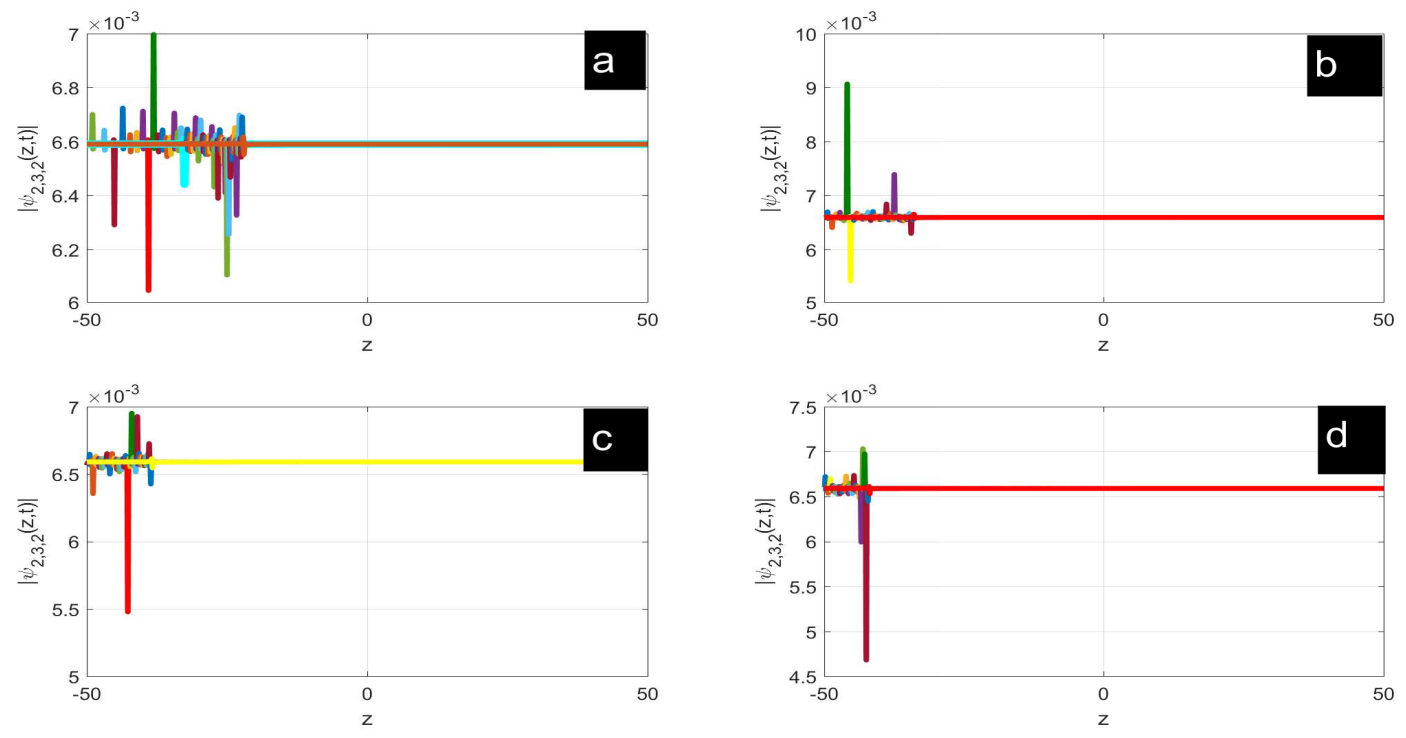

Figure 7: Breather-like soliton evolution $\left|\delta \psi_{2,3,2}(z, t)\right|^{2}$ with the effects of the CFP (a) [ $\left.\beta=0.1\right]$, (b) $[\beta=0.12],(\mathrm{c})[\beta=0.13],(\mathrm{d})[\beta=0.14]$ at $B=e, \alpha=0.01, \epsilon=-0.02, s=0.25, H_{4}=0.095, \theta=$ $0.1, H_{2}=0.94, \gamma=2.045, A_{0}=0.06, A_{1}=0.05, \tau=1, g=0.04, h=0.0007$.

- Case 5: For $H_{0}=H_{1}=0$, and $H_{2}>0, \tau= \pm 1$, using Eqs.(31-32) respectively, is revealed as

$$
\begin{aligned}
& \psi_{2,5,1}(z, t)=\sqrt{\frac{g h A_{1} H_{2} H_{3} \operatorname{sech}_{B}^{2}\left(\tau \sqrt{2 H_{2}} \frac{\left(\frac{t \beta}{\beta}-u z\right)}{2}\right)}{H_{2} R_{4}\left(1-\tanh _{B}\left(\tau \sqrt{2 H_{2}} \frac{\left(\frac{t^{\beta}}{\beta}-u z\right)}{2}\right)\right)^{2}-H_{3}^{2}}} e^{i\left(C\left(\frac{t^{\beta}}{\beta}-u z\right)-\kappa z\right),} \\
& \psi_{2,5,2}(z, t)=\sqrt{A_{0}+\frac{g h A_{1} H_{2} H_{3} \operatorname{sech}_{B}^{2}\left(\tau \sqrt{2 H_{2}} \frac{\left(\frac{t^{\beta}}{\beta}-u z\right)}{2}\right)}{H_{2} H_{4}\left(1-\tanh _{B}\left(\tau \sqrt{2 R_{2}} \frac{\left(\frac{t^{\beta}}{\beta}-u z\right)}{2}\right)\right)^{2}-H_{3}^{2}}} e^{i\left(C\left(\frac{t^{\beta}}{\beta}-u z\right)-\kappa z\right)} .
\end{aligned}
$$

the chirp components is set out as

$$
\begin{aligned}
& \delta \omega_{2,5,1}(z, t)=-\frac{(3 \gamma+2 \epsilon)}{4 \alpha \vartheta} \frac{g h A_{1} H_{2} H_{3} \operatorname{sech}_{B}^{2}\left(\tau \sqrt{2 H_{2}} \frac{\left(\frac{t^{\beta}}{\beta}-u z\right)}{2}\right)}{H_{2} R_{4}\left(1-\tanh _{B}\left(\tau \sqrt{2 H_{2}} \frac{\left(\frac{t^{\beta}}{\beta}-u z\right)}{2}\right)\right)^{2}-H_{3}^{2}}+\frac{1}{2 \alpha \vartheta^{2}}, \\
& \delta \omega_{2,5,2}(z, t)=-\frac{(3 \gamma+2 \epsilon)}{4 \alpha \vartheta}\left[A_{0}+\frac{g h A_{1} H_{2} H_{3} \operatorname{sech}_{B}^{2}\left(\tau \sqrt{2 H_{2}} \frac{\left(\frac{t^{\beta}}{\beta}-u z\right)}{2}\right)}{H_{2} H_{4}\left(1-\tanh _{B}\left(\tau \sqrt{2 R_{2}} \frac{\left(\frac{t^{\beta}}{\beta}-u z\right)}{2}\right)\right)^{2}-H_{3}^{2}}\right]+\frac{1}{2 \alpha \vartheta^{2}} .
\end{aligned}
$$

\section{Modulation Instability}

\subsection{Linear stability analysis}

We investigate the MI growth rate with the effects of the FTP. MI is the processus which show the behavior of the $\mathrm{CW}$ with tiny perturbations during the confrontation between nonlinearity and dispersion terms. Many studies have been done to show the effects of optical parameters on MI such as the normal and anomalous dispersion regime combined with SS, Self-phase modulation (SPM), Cross Phase modulation (XPM) in birefringent OF, Higher-Order dispersions (HOD) and so on. [7-11, 15, 16, 26, 27, 29, 31, 40]. It is worth to 
mention that the effects of CFP have been recently set out on MI growth rate associated to Third-Order dispersions (TOD). These results have shown the formation of instability zones (instable CW) when the value of the CFP increases and for small value the stability zones. In this work, we fixed the value of the NLSE parameters and varying the CFP to stress the behavior of MI gains spectra. We use the linearizing technic, to establish the dispersion relation and the MI gain. We assume the $\mathrm{CW}$ with small disturbances as solution of the set Eq.(12)

$$
\psi(z, t)=\left(P_{0}+F(z, t)\right) e^{i k z}
$$

Here $D(z, t)$ is the tiny perturbation introduced to examine the MI gain. Inserting Eq.(45) into the set of coupled Eq.(12), the linearizing technic gives

$$
i F_{z}+\frac{\alpha}{(2 \beta)} \frac{\partial^{2 \beta} F}{\partial t^{2 \beta}}+\frac{i \epsilon P^{2}}{\beta}\left(\frac{\partial^{\beta} F}{\partial t^{\beta}}+\frac{\partial^{\beta} F^{*}}{\partial t^{\beta}}\right)+\frac{i \gamma P^{2}}{\beta}\left(2 \frac{\partial^{\beta} F}{\partial t^{\beta}}+\frac{\partial^{\beta} F^{*}}{\partial t^{\beta}}\right)+s P^{2}\left(2 F+F^{*}\right)+\theta P^{4}\left(3 F+2 F^{*}\right)=0,
$$

$F(z, t)^{*}$ is the complex conjugated of $F(z, t)$. We consider the solution of Eq.(46) in the form of

$$
F(z, t)=f_{1} e^{i(K z-\Omega t)}+f_{2} e^{-i(K z-\Omega t)},
$$

where $f_{1}$ and $f_{2}$ are the disturbance amplitudes, while $K$ and $\Omega$ are wave number and angular frequency of the MI respectively. Making some algebraic manipulations, the dispersion relation with second-order polynomial gives

$$
m_{2} K^{2}+m_{1} K+m_{0}=0
$$

where

$$
\begin{aligned}
& m_{2}=-((2 \beta))^{2}(\beta)^{2}, \varepsilon_{1}=e^{-i \alpha \pi \operatorname{sign}(\Omega) / 2}, \varepsilon_{2}=e^{i \alpha \pi \operatorname{sign}(\Omega) / 2}, \\
& m_{1}=(|\Omega|)^{2 \beta}\left((2 \beta)(\beta)^{2} \alpha\left(\epsilon_{1}-\epsilon_{2}\right)\left(\epsilon_{1}+\epsilon_{2}\right)\right)+(|\Omega|)^{\beta}\left(((2 \beta))^{2} \beta P^{2} i\left(\epsilon_{1}-\epsilon_{2}\right)(2 \gamma+\epsilon)\right), \\
& m_{0}=(|\Omega|)^{4 \beta}\left((\beta)^{2} \alpha^{2} \epsilon_{1}^{2} \epsilon_{2}^{2}\right)+(|\Omega|)^{3 \beta}\left((2 \beta) \beta P^{2} \epsilon_{1} \epsilon_{2} \alpha i\left(\epsilon_{1}+\epsilon_{2}\right)(2 \gamma+\epsilon)\right)+(|\Omega|)^{2 \beta}(2 \beta) P^{2}\left(2(\beta)^{2} \epsilon_{2}{ }^{2} \alpha s\right) \\
& -(|\Omega|)^{2 \beta}\left((2 \beta) P^{2}\left(-3(\beta)^{2} P^{2} \epsilon_{1}{ }^{2} \alpha \theta-3(\beta)^{2} P^{2} \epsilon_{2}{ }^{2} \alpha \theta+2(2 \beta) P^{2} \epsilon_{1} \epsilon_{2} \epsilon \gamma+3(2 \beta) P^{2} \epsilon_{1} \epsilon_{2} \gamma^{2}-2(\beta)^{2} \epsilon_{1}{ }^{2} \alpha s\right)\right) \\
& +(|\Omega|)^{\beta}\left(((2 \beta))^{2} \beta P^{4} i\left(\epsilon_{1}+\epsilon_{2}\right)\left(\epsilon \theta P^{2}+4 \gamma \theta P^{2}+\epsilon s+3 \gamma s\right)\right)+\left(((2 \beta))^{2}(\beta)^{2} P^{4}\left(5 \theta P^{2}+3 s\right)\left(\theta P^{2}+s\right)\right)
\end{aligned}
$$

In plots figure 8 (a, b, c, d), we show the effects of the CFP on the MI gain in anomalous dispersion regime. We fixed the values of the model parameters. For $\beta=0.1$, it is observed the appearance of one side lobe. After increasing the value of the CFP to $\beta=0.3$, the side lobe decreases and the MI growth rate amplitude decreases. Thereafter, we increase the value of the CFP to $\beta=0.8$, the side lobe tends to vanish and the MI band shrinks, while the amplitude increases. Now, in normal dispersion regime, we observe the formation of tiny instability zones for $\beta=0.2$ (see figure 9 (a)). By increasing the value of the CFP, in figure 9 (b, c,d) we observed that the stability zones increase and the amplitude of the MI gain increases. To confirm these results, we will use numerical study in the next section by using pseudospectral technic associated with the fourth-order Runge-Kutta. 

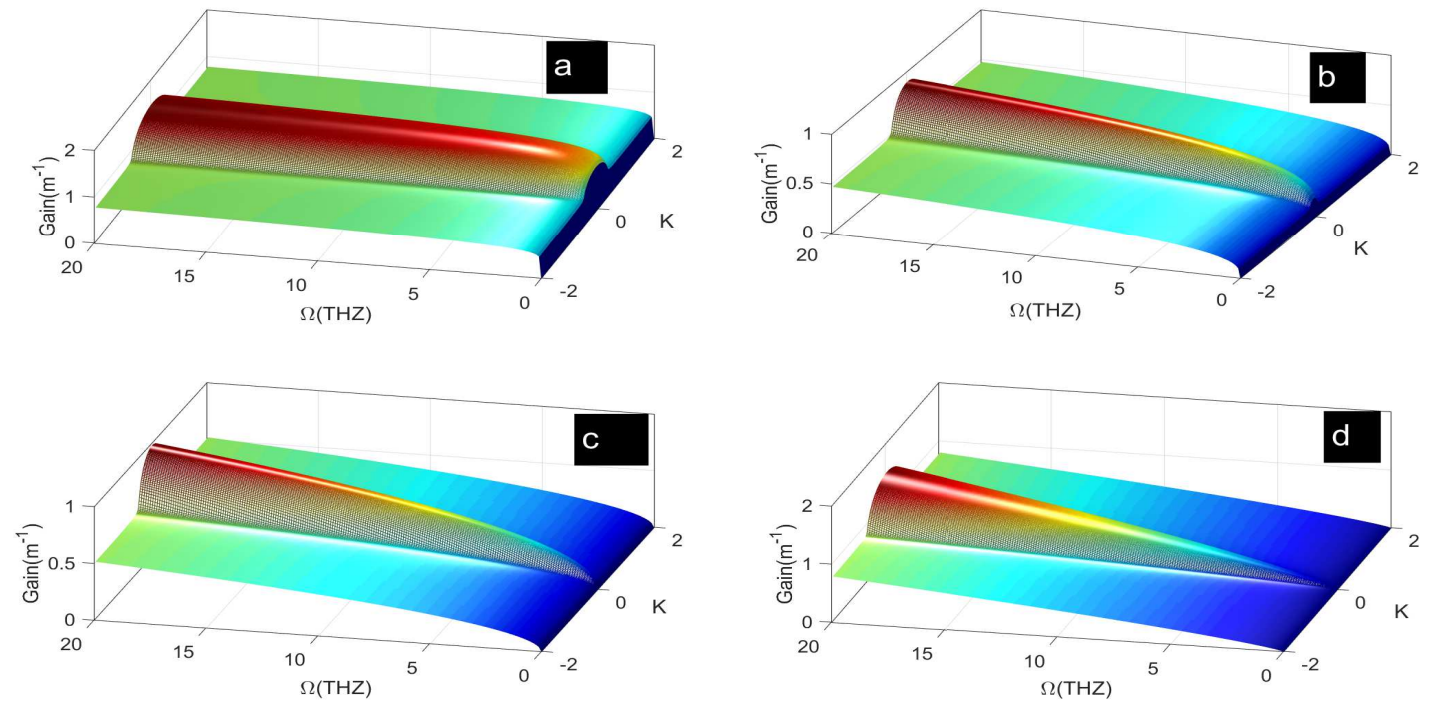

Figure 8: MI growth rate with the variation of the CFP in normal dispersion regime (a) $[\beta=0.1]$, (b) $[\beta=0.3]$, (c) $[\beta=0.5]$ and (d) $[\beta=0.8]$ at $P_{0}=0.25, \alpha=0.01, \epsilon=0.8, s=0.25, \theta=0.45, \gamma=0.06$.
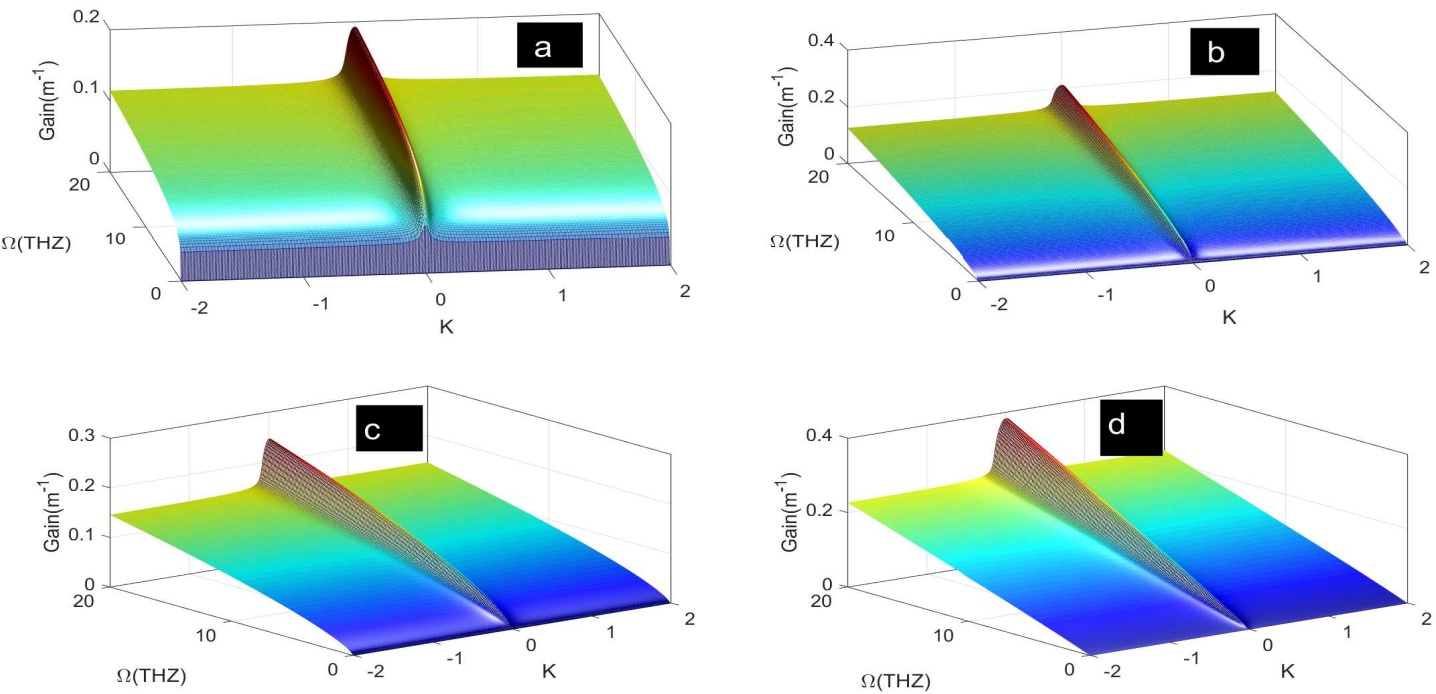

Figure 9: MI growth rate with the variation of the CFP in anomalous dispersion regime (a) $[\beta=0.2]$, (b) $[\beta=0.6],(\mathrm{c})[\beta=0.7]$ and (d) $[\beta=0.85]$ at $P_{0}=0.25, \alpha=-0.1, \epsilon=0.8, s=0.25, \theta=0.45, \gamma=0.06$. 


\section{Numerical simulation}

We numerically investigate the robustness of the above analytical results of the NLSE describing the polarization mode in OF. We firstly used Eq.(24) and Eq.(28) with their corresponding chirp components to confirm the effects of the CFP by fixing the MP. Thereafter, to verify the prediction made on the MI growth rate.

\subsection{Effects of the fractional parameter on numerical soliton solutions}

We use the Split-step Fourier which is based on the pseudospectral technic associated with the fourth-order Runge-Kutta integration alongside time direction to verify the effects of the CFP and stability of the obtained analytical results. We use for this purpose the discrete Fourier mechanism to assess the spatial derivative. We set the Fourier transform of NLSE Eq.(12) with FT order as

$$
\begin{aligned}
& \hat{\psi}_{z}-i \alpha(i \omega)^{2 \beta} \hat{\psi}=\mathcal{F}\left[s\left(|\psi(z, t)|^{2} \psi(z, t)\right)+i \gamma D_{t}^{\beta}\left(|\psi(z, t)|^{2} \psi(z, t)\right)\right. \\
& \left.+i \epsilon \psi(z, t) D_{t}^{\beta}\left(|\psi(z, t)|^{2}\right)+\theta|\psi(z, t)|^{4} \psi(z, t)\right],
\end{aligned}
$$

with

$$
\hat{q}=\hat{\psi} e^{-i \alpha(i \omega)^{2 \beta} z}
$$

We consider the fourth-order Runge-Kutta with time step $\Delta t=0.001$. The following set of analytical parameters of the model are used: $A_{0}=-0.06, A_{1}=0.5, H_{2}=1.84, H_{4}=-0.09, h=0.0078, g=$ $0.042, s=0.25, \theta=0.1, \alpha=0.01, \gamma=0.045, \epsilon=-0.02$. The assumption consists in keeping the values of the model and NGEAM parameters fixed and then varying the CFP to observe the behavior of the optical soliton during propagation.

In figure 10 we show the behavior of the $\mathrm{W}$-shaped profile with the variation of CFP in anomalous dispersion regime. The constraint conditions are the same on the analytical results (i.e. $H_{2}<0$ and $\left.H_{4}>0\right)$. We notice that when the CFP increases, the $\mathrm{W}$-shaped profile propagated from right to left without any deformation and maintains shaped. However, in figure 11 (a, b) it is shown the propagation of the corresponding chirp of bright optical soliton in anomalous dispersion regime $(\alpha=-0.01)$ with fixed value of the model parameter and variation of the CFP. In figure 11(a, b) we display the chirp bright for $\beta=0.25$ and then when we increase the value of the CFP to $\beta=0.6$, its position changes. In the meantime, in figure 13 (a, b, c, d) we show the corresponding chirp anti-kink-like soliton wit the variation of the FP and fixed value of the MP. The constraint condition remain the same in figure 11. At the end we notice that: (i) the FD parameter induces the movement of the optical soliton; (ii) optical soliton propagates while retaining its amplitude; (iii) the presence of the FD parameter stimulates energy at the soliton to allow it to propagate in the optical medium. 

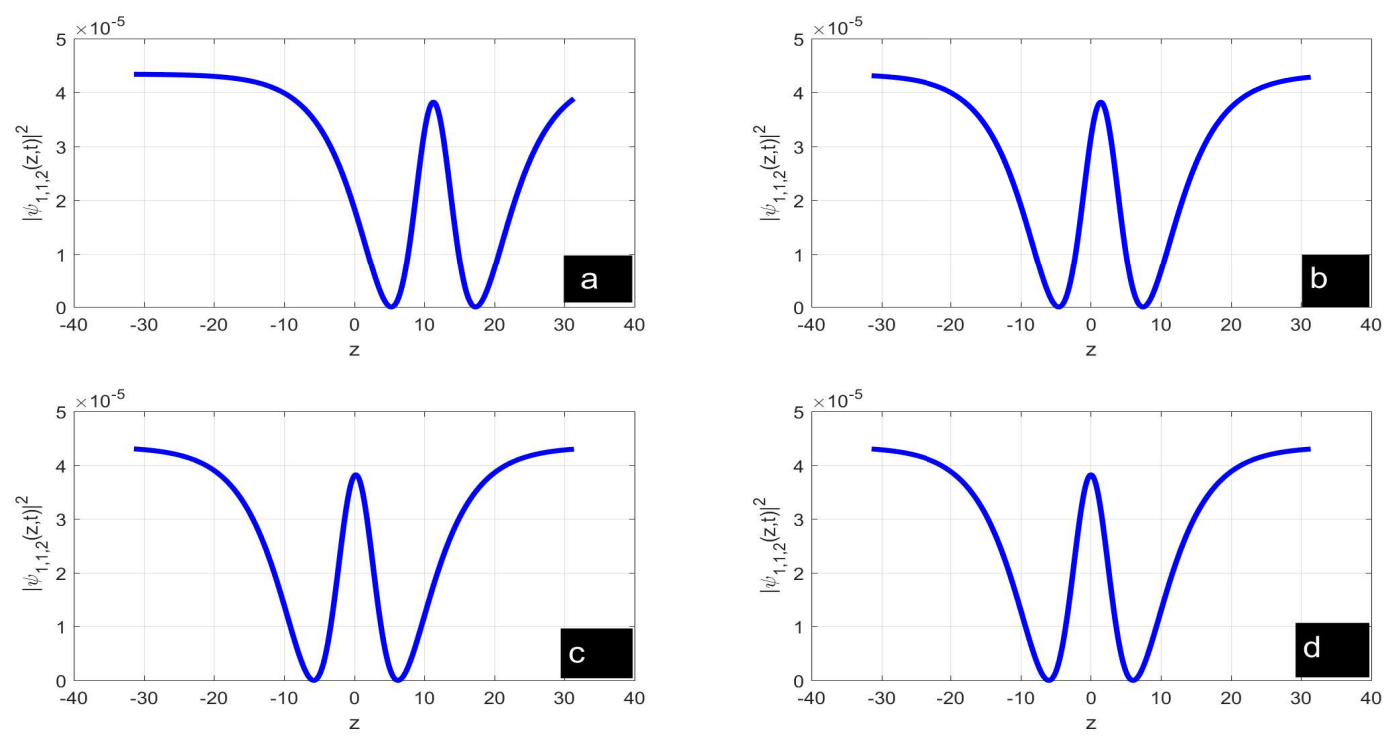

Figure 10: Propagation of the W-shaped profile of $\left|\psi_{1,1,2}(z, t)\right|^{2}$ with the effects of CFP in normal dispersion regime (a) $[\beta=0.2]$, (b) $[\beta=0.4]$, (c) $[\beta=0.6]$ and (d) $[\beta=0.85]$.
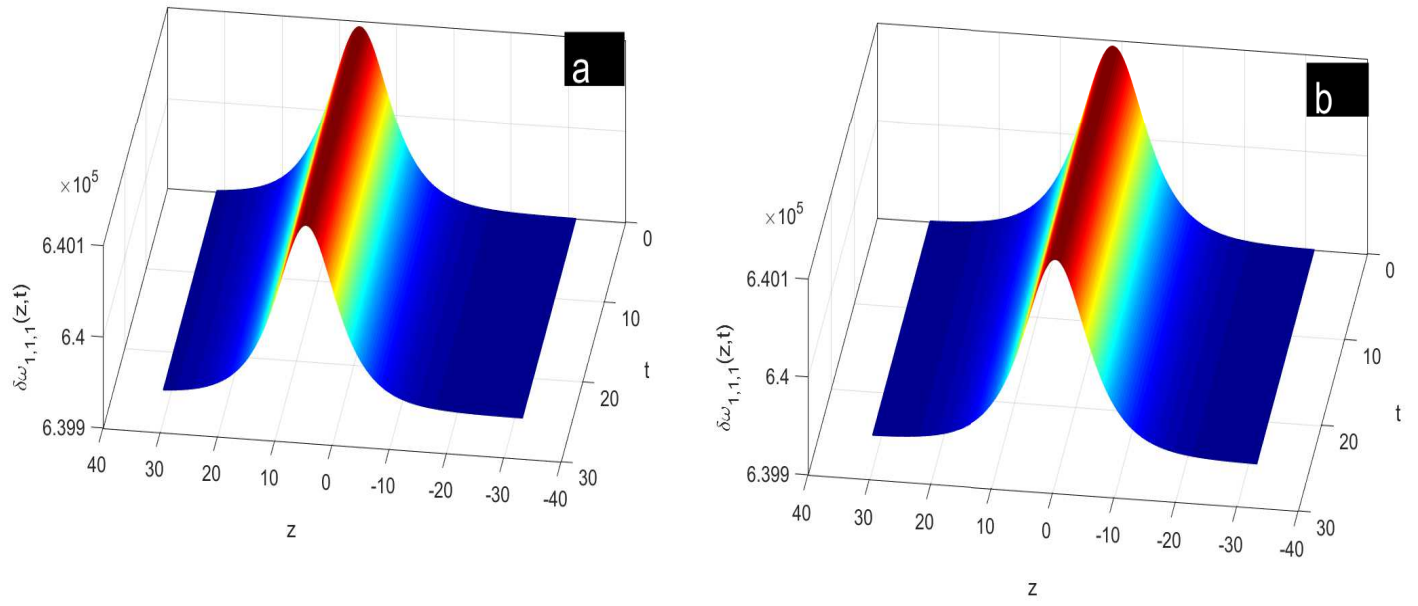

Figure 11: Propagation of the bright chirp soliton profile of $\delta \omega_{1,1,2}(z, t)$ with the effects of CFP in normal dispersion regime (a) $[\beta=0.25]$, (b) $[\beta=0.4]$. 

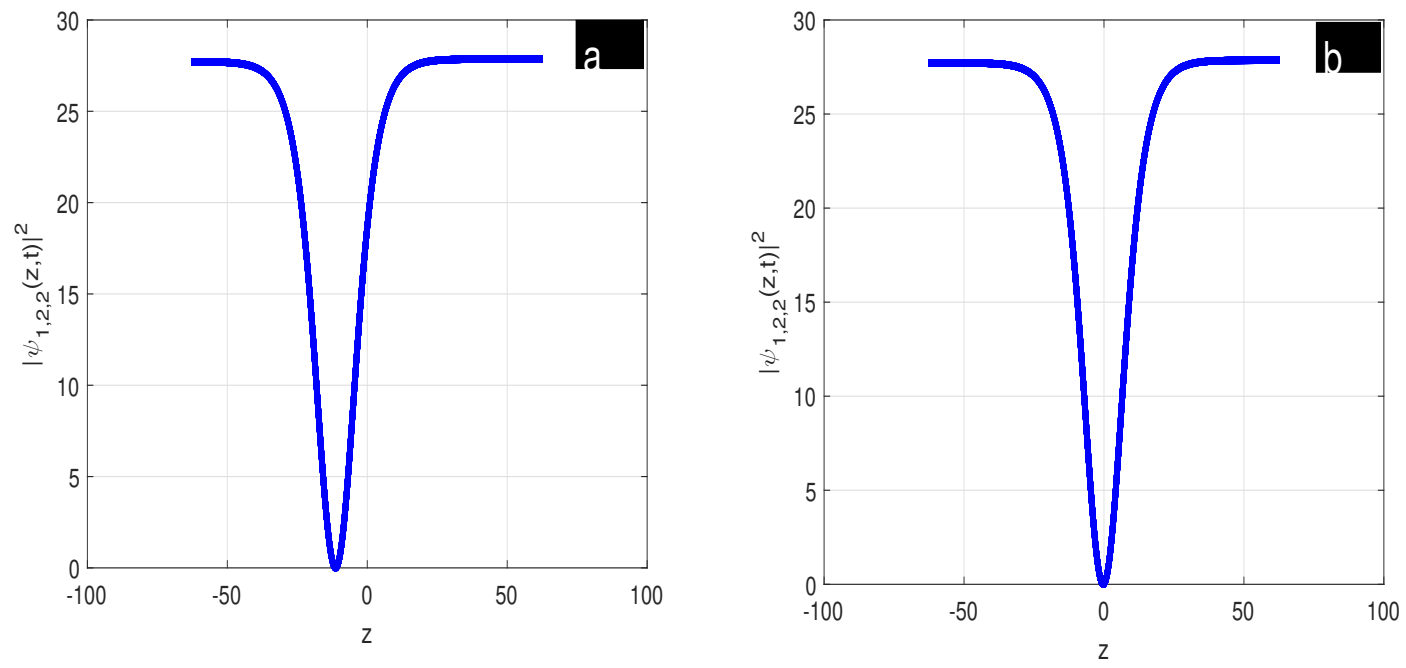

Figure 12: Propagation of dark soliton solutions $\psi_{1,2,2}(z, t)$ with the effects of CFP in normal dispersion regime (a) $[\beta=0.2]$ and (b) $[\beta=0.6]$.
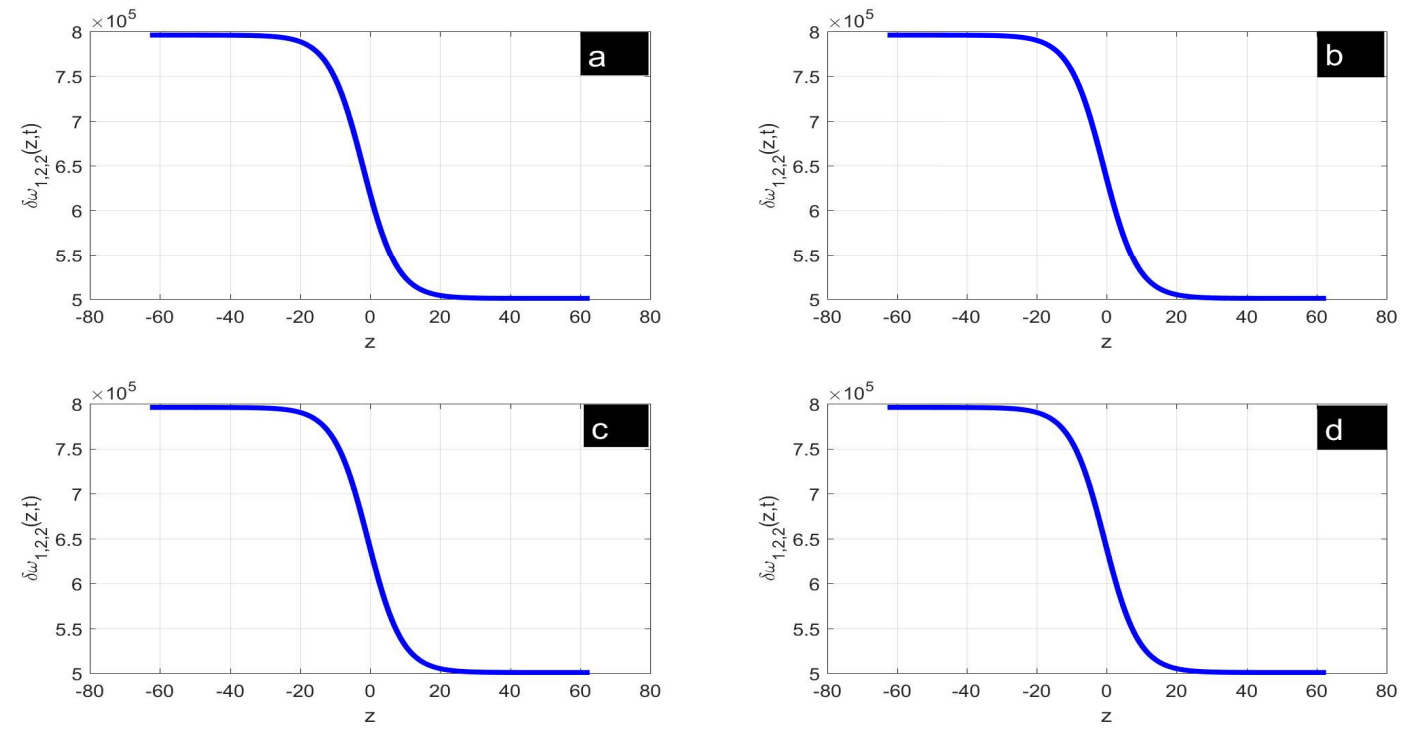

Figure 13: Propagation of anti-kink-like soliton of $\delta \omega_{1,2,2}(z, t)$ with the effects of CFP in normal dispersion regime (a) $[\beta=0.2]$, (b) $[\beta=0.6]$, (c) $[\beta=0.85]$ and (d) $[\beta=1]$. 

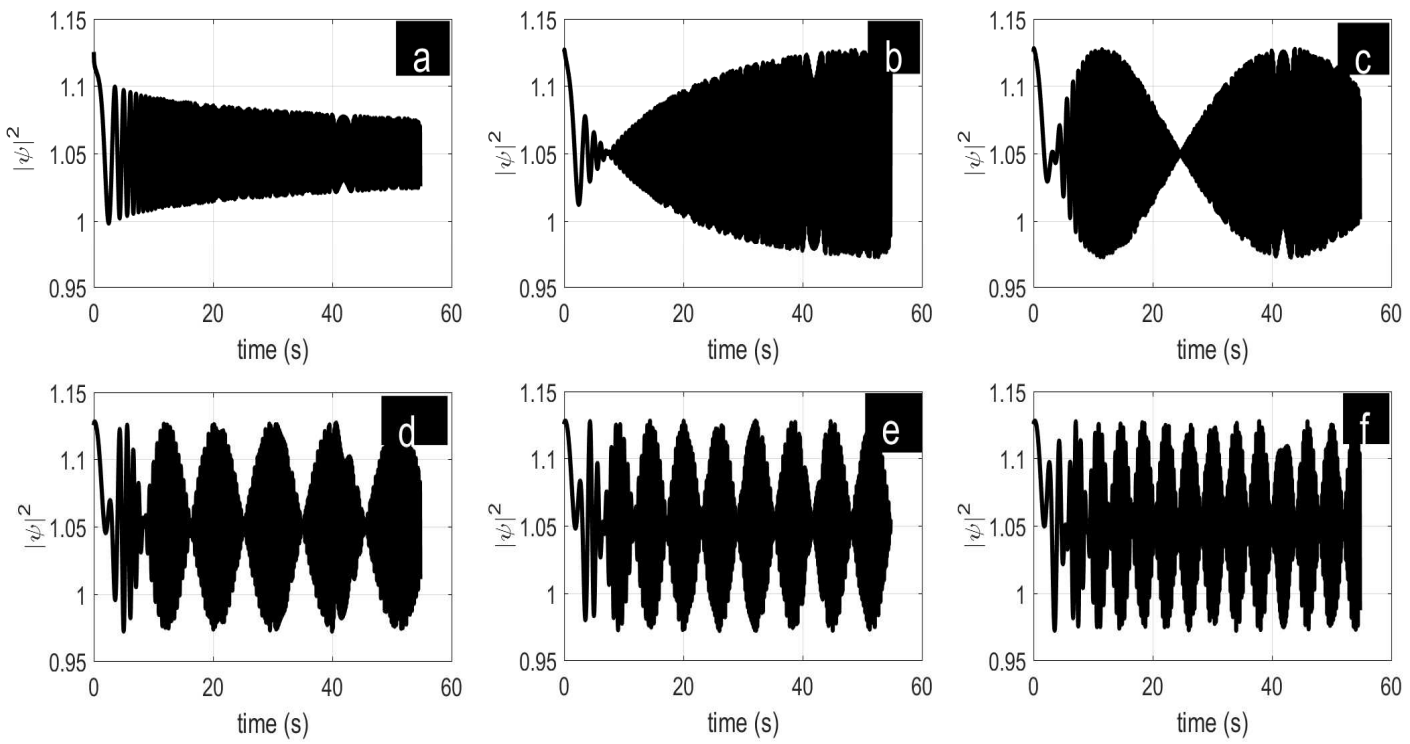

Figure 14: Numerically evolution of breather-like soliton $|\psi|^{2}$ Eq.(51) in anomalous dispersion regime with the effects of CFP top panel (a) $\beta=0.1$, (b) $\beta=0.3$, (c) $\beta=0.5$, (d) $\beta=0.75$, (e) $\beta=0.85$ and (f) $\beta=0.95$ the breather-like soliton .

\subsection{Numerical analysis of MI growth rate}

We examine the MI growth rate by using the numerical simulation in this section. Let's assume the initial condition in the form of the perturbed plane wave as follows:

$$
\psi(z, 0)=\left(P_{0}+\xi \cos \left(K_{0} z\right)\right) e^{i k z} .
$$

To moreover examine the stability or instability zones, we set $P_{0}=1.5, \xi=0.001, \alpha=0.1, \theta=0.5, \gamma=$ $0.45, \epsilon=0.02, k=0.5$. The time step is $\Delta t=0.001$ and the grid with $N=2^{10}$ nodes. In plots figure 14, the evolution of the $|\psi|^{2}$ anomalous dispersion regime $(\alpha=-0.8)$ with the effects of the CFP and the excitation parameter $K_{0}=1.05$. We realize that the amplitude does not increase and the maximum value is 1.12 despite the increasing of the CFP. However, it is observed the formation of breather like-soliton when we increase the value of the CFP (see figure $14(\mathrm{c}, \mathrm{d}, \mathrm{e}, \mathrm{f})$ ). Otherwise, for $\beta=0.85$ in figure (14 (f)), we observe chaotic breathing, which means that the whole system vibrates and therefore the system becomes unstable and difficult to control. In figure 15, we use the same time step in normal dispersion regime $(\alpha=0.8)$, we observe that the breather like-soliton propagates very fast when the CFP increases and the maximum amplitude does not grow up to 1.12 (see figure 15 (a, b, c, d)). It performs harsh oscillations in the range of [0.97-1.12], which confirms the stability of the system.

\section{Conclusion and Remarks}

The present work shows the effects of the CFP on optical soliton solutions and MI growth rate. We employ the NGEAM to deal with three families of optical soliton solutions and their corresponding chirp such as 

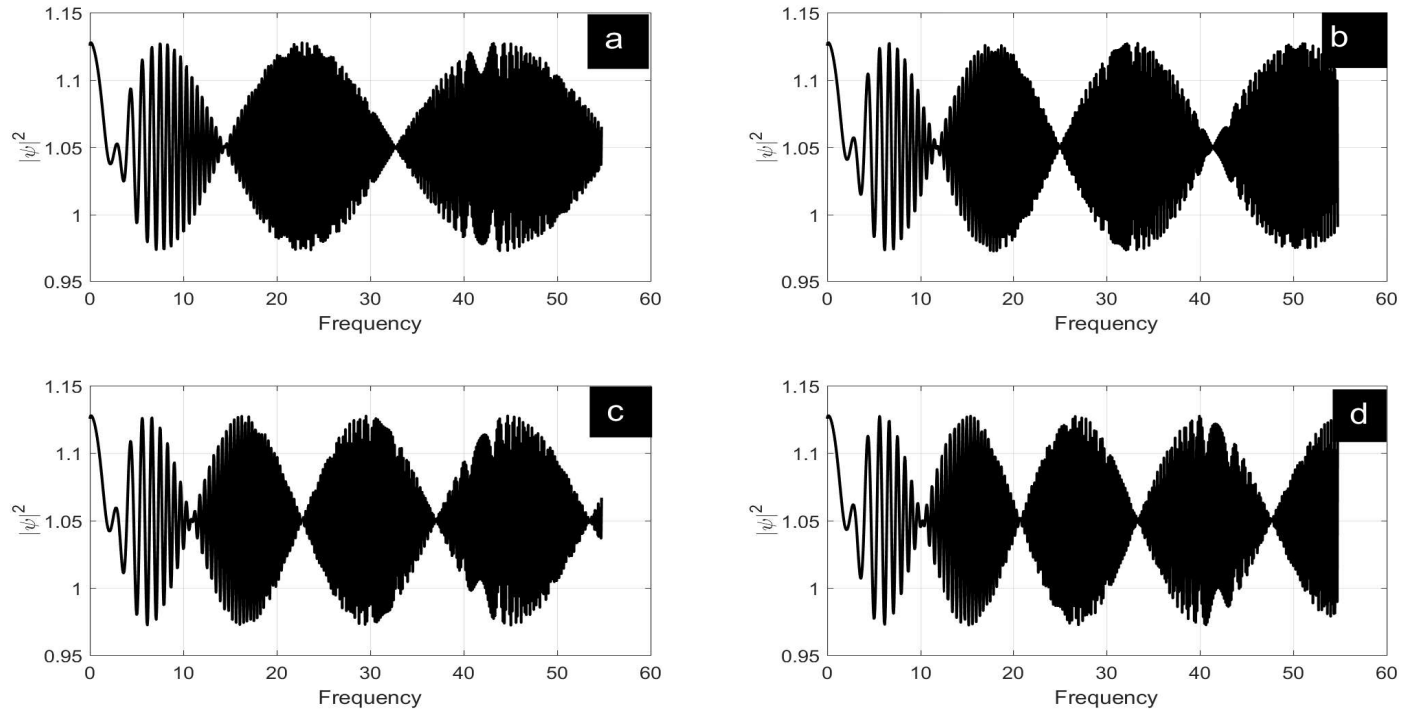

Figure 15: Numerically evolution of $|\psi|^{2}$ Eq.(51) in normal dispersion regime with the effects of CFP top panel (a) $\beta=0.6$, (b) $\beta=0.65$ and bottom panel (c) $\beta=0.66$ (d) $\beta=0.67$ with the excitation wavenumber $K_{0}=1.05$.

bright, dark and the combined bright-dark optical solitons. Through out figures 1, 2, 3, 4 and 5, we show the FTP effects on analytical results. We examine the MI growth rate with the effects of the FTP. It is observed that when the CFP induces instability zones and increases MI bands. For small values of the FT and fixed values of the MP, the MI growth rate (unstable continuous waves). We use numerical simulation to corroborate and confirm the stability and the effective effects of the CFP on the obtained analytical results. We notice that when the CFP increases, W-shaped profile can propagate very fast and maintains stable shape. Additionally, the effects of the CFP on the corresponding chirp of the dark optical soliton have been obtained. Through out numerical simulation, we show the effects of the fractional order on MI gain. We observe the formation of breather-like soliton in normal and anomalous dispersion regime. In the case of small values of the CFP, we obtain stable breather-like solitons which are formed during strong oscillations with stable amplitude and shape during the propagation. Compared these results with refs. [15, 17, 18, 26, 27, 33], new behavior of optical soliton solutions and W-shaped profile have been obtained. Owing to the CFP, these results could pave the way to such applications in OF and we will add 4WM terms to analyze the behavior of the MI gain for feature works.

\section{Competing interests}

The authors declare no competing interests. 


\section{Data availability statements}

The datasets generated during and/or analysed during the current study are available from the corresponding author on reasonable request.

\section{References}

[1] A. Yusuf, M. Inc, D. Baleanu, Optical solitons with M-truncated and beta derivatives in nonlinear optics. Frontiers in Physics, 7 (2019) 126.

[2] C. A. S. Gómez, A. Jhangeer, H. Rezazadeh, R. A. Talarposhti, A. Bekir, Closed Form Solutions of the Perturbed Gerdjikov-Ivanov Equation With Variable Coefficients, East Asian Journal on Applied Mathematics 11 (2021) 207.

[3] A. Jhangeer, N. Raza, H. Rezazadeh A. R. Seadawy, Nonlinear self-adjointness, conserved quantities, bifurcation analysis and travelling wave solutions of a family of long-wave unstable lubrication model, Pramana-J. Phys. (2020) 94:87.

[4] A. Houwe, S. Abbagari, Y. Salathiel, M. Inc, S. Y. Doka, K. Timoléon Crépin \& S. Y. Doka, Complex traveling-wave and solitons solutions to the Klein-Gordon-Zakharov equations, Results in Physics. 17 (2020) 103127.

[5] A. Jhangeer, H. Rezazadeh A. R. Seadawy, A study of travelling, periodic, quasiperiodic and chaotic structures of perturbed Fokas-Lenells model, Pramana-J.Phys. (2021) 95:41.

[6] A. Jhangeer, A. Hussainb, M. Junaid-U-Rehmanb, D. Baleanuc, M. B. Riaz, Quasi-periodic, chaotic and travelling wave structures of modified Gardner equation, Chaos Solitons and Fractals 143 (2021) 110578.

[7] J. H. Li, K. S. Chiang, K. W. Chow, Modulation instabilities in two-core optical fibers, J. Opt. Soc. Am. B 28 (2011) 1693-1701.

[8] T. Tanemura, Y. Ozeki, K. Kikuchi, Modulational instability and parametric amplification induced by loss dispersion in optical fibers, Phys. Rev. Lett. 93 (2004) 163902.

[9] S. G. Murdoch, R. Leonhardt, J. D. Harvey, Polarization modulation instability in weakly birefringent fibers, Opt. Lett. 20 (1995) 866-868.

[10] J. E. Rothenberg, Modulational instability for normal dispersion, Phys. Rev. A 42 (1990) 682-685.

[11] P. D. Drummond, T. A. B. Kennedy, J. M. Dudley, R. Leonhardt, J. D. Harvey, Cross-phase modulationalinstability in highbirefringence fibers, Opt. Commun.78 (1990) 137-142.

[12] I. Podlubny, Fractional Differential Equations, Academic Press, San Diego, 1999.

[13] A. Abdon, B. Dumitru, New fractional derivatives with nonlocal and non-singular kernel: theory and application to heat transfer model, Therm. Sci. 20(2) (2016) 763-769. 
[14] R. Khalil, M. Al Horani, A. Yousef, M. Sababheh, A new definition of fractional derivative, J. Comput. Appl. Math. 264 (2014) 65-70.

[15] A. Houwe, S. Abbagari, K. S. Nisar, M. Inc \& S. Y. Doka, Influence of fractional time order on Wshaped and Modulation Instability gain in fractional Nonlinear Schrödinger Equation, Results in Physics (2021) 104556.

[16] A. Houwe, S. Abbagari, S. Y. DOKA, M. Inc \& T. B. Bouetou, Clout of fractional time order and magnetic coupling coefficients on the soliton and modulation instability gain in the Heisenberg ferromagnetic spin chain. Chaos, Solitons and Fractals 151 (2021) 111254.

[17] D. Bienvenu, B. Gambo \& Mama Nsangou, Effects of fractional temporal evolution on chirped soliton solutions of the Chen-Lee-Liu equation. Phys. Scr. 96 (2021) 105215.

[18] M. Eslami, E. K. Hosseini, E. M. Matinfar, E. M. Mirzazadeh, E. M. Ilie \& E. J. F. Gomez-Aguilar, A nonlinear Schrdinger equation describing the polarization mode and its chirped optical solitons, Optical and Quantum Electronics 53 (2021) 314.

[19] E. Tala Tebue, Z. I. Djoufack, S. B. Yamgoua, A. Kenfack Jiotsa \& T. C. Kofane, Chirped soliton in optical medium, Optical and Quantum Electronics 51 (2019) 7.

[20] M. Desaix, L. Helczynski, D. Anderson \& M. Lisak, Propagation properties of chirped soliton pulses in optical nonlinear Kerr media. Phys. Rev. E 65 (2002)056602.

[21] N. Raza, A. R. Seadawy, A. J. Asma Rashid Butt, S. Arshed, Dynamical behavior of micro-structured solids with conformable time fractional strain wave equation, Physics Letters A, 384(27) (2020) 126683.

[22] M. Kaplan, A. Bekir, M. N. Ozer, A simple technique for constructing exact solutions to nonlinear differential equations with conformable fractional derivative, Opt Quant Electron 49(2017) 266.

[23] M. Kaplan, Applications of two reliable methods for solving a nonlinear conformable time-fractional equation, Opt Quant Electron 49 (2017) 312.

[24] Susanto H., Malomed B.A., Embedded solitons in second-harmonic-generating lattices, Chaos, Solitons and Fractals 142 (2021) 110534.

[25] Rao J.G., Malomed B.A., Cheng Y., He J.S., Dynamics of interaction between lumps and solitons in the Mel'nikov equation, Commun. Nonlinear Sci. Numer. Sim. 91 (2020) 105429.

[26] A. Houwe, S. Abbagari, S. Nestor, M. Inc, Mir Sajjad Hashemi, G. Betchewe \& S. Y. Doka, Optical soliton and weierstrass elliptic function management to parabolic law nonlinear directional couplers and modulation instability spectra, Optical and Quantum Electronics 53(8)(2021) 417.

[27] A. Souleymanou, A, A. Houwe, S. P. Takougoum Mukam, H. Rezazadeh, M. Inc, S. Y. Doka \& T. B. Bouetou, Optical solitons to the nonlinear Schrödinger equation in metamaterials and modulation instability, European Physical Journal Plus 136(7)(2021) 710. 
[28] A. Souleymanou, A. Houwe, H. Rezazadeh, A. Bekir \& S. Y. Doka, Solitary wave solutions in two-Core optical fibers with coupling-coefficient dispersion and Kerr nonlinearity, Revista Mexicana de Fisica 67(3) (2021) 369 .

[29] A. Souleymanou, , A. Houwe, D. Douvagai, S. Y. Doka \& T. C. Kofane, M-shape and W-shape bright incite by the fluctuations of the polarization in a-helix protein, Physica Scripta 96(8) (2021).

[30] A. Houwe, S. Abbagari, B. Almohsen, G. Betchewe, M. Inc \& S. Y. Doka. Chirped solitary waves of the perturbed Chen-Lee-Liu equation and modulation instability instability in optical monomode fibres. Optical and Quantum Electronics. 53(2021) 286.

[31] A. Houwe et al. Survey of third- and fourth-order dispersions including ellipticity angle in birefringent fibers on W-shaped soliton solutions and modulation instability analysis, European Physical Journal Plus 136 (4) (2021) 357.

[32] M. J Ablowitz \& T. P. Horikis, Rogue waves in birefringent optical fibers: elliptical and isotropic fibers, J. Opt. 19 (2017) 065501.

[33] A. Houwe., S. Abbagari , M. Inc, G. Betchewe, S. Y. Doka \& K. Timoléon Crépin, Chirped solitons in discrete electrical transmission line, Results in Physics. 18 (2020) 103188.

[34] S. Abbagari, Alphonse H. Alphonse, S. Youssoufa, D. Douvagai, Y. Chu, ,M. Inc, H. Rezazadeh, S.Y. Doka, Analytical survey of the predator-prey model with fractional derivative order, AIP Advances 11(3), 035127 (2021).

[35] A. Souleymanou, A. Korkmaz, H. Rezazadeh, S. P. Takougoum Mukam, A. Bekir, Soliton Solutions in different classes for the Kaup-Newell model equation, Modern Physics Letters B. 34, 2050038(2019).

[36] A. Souleymanou, K. K. Ali, H. Rezazadeh, M. Eslami, M. Mirzazadeh, A. Korkmaz, The propagation of waves in thin-film ferroelectric materials, Pramana. J. Phys. 93, 27(2019).

[37] S. P. T. Mukam, A. Souleymanou, V. K. Kuetche, T. B. Bouetou, Generalized Darboux transformation and parameter-dependent rogue wave solutions to a nonlinear Schrodinger system,Nonlinear Dynamics. 93, 56(2018).

[38] A. Souleymanou, A, A. Houwe, S. P. Takougoum Mukam, H. Rezazadeh, M. Inc, S. Y. Doka, T. B. Bouetou, Optical solitons to the nonlinear Schrödinger equation in metamaterials and modulation instability, European Physical Journal Plus 136(7)(2021) 710;

[39] H. Yepez-Martinez, H. Rezazadeh, A. Souleymanou, S. P. T. Mukam, M. Eslami, V. K. Kuetche A. Bekir, The Extended Modified Method Applied to Optical Solitons solutions in Birefringent Fibers with weak nonlocal nonlinearity and four wave mixing, Chinese Journal of Physics 58 (2019) 137-150.

[40] S. Abbagari, A. Houwe, S. Y. Doka, T. B. Bouetou, M. Inc, K. T. Crepin, W-shaped profile and multiple optical soliton structure of the coupled nonlinear Schrödinger equation with the four-wave mixing term and modulation instability spectrum, Physics Letters A. 418 (2021) 127710. 